\title{
Asynchronous laser transponders for precise interplanetary ranging and time transfer
}

\author{
John J. Degnan \\ Geoscience Technology Office, Code 920.3, Laboratory for Terrestrial Physics, \\ NASA Goddard Space Flight Center, Greenbelt, MD 20771, USA
}

\begin{abstract}
Satellite laser ranging (SLR) and lunar laser ranging (LLR) systems are single-ended instruments, i.e. they measure the roundtrip transit time of a laser pulse to a passive optical reflector. Since such single-ended systems are incapable of ranging beyond the Moon to the planets, we consider the feasibility of a two-way asynchronous (i.e. independently firing) interplanetary laser transponder pair, capable of decimeter ranging and subnanosecond time transfer from Earth to a spacecraft anywhere within the inner Solar System. After introducing the transponder link equation and the concept of "balanced" transponders, we describe how range and time can be transferred between terminals, and preview the potential advantages of photon counting asynchronous transponders for interplanetary applications. We then develop mathematical models for the various sources of noise in an interplanetary transponder link including planetary albedo, solar or lunar illumination of the local atmosphere, and laser backscatter off the local atmosphere. After introducing the key engineering components of an interplanetary laser transponder, we develop an operational scenario for the acquisition and tracking of the opposite terminal. We then use the theoretical models of the previous sections to perform an Earth-Mars link analysis over a full synodic period of 780 days under the simplifying assumption of coaxial, coplanar, circular orbits. We demonstrate that, using slightly modified versions of existing space and ground based laser systems, an Earth-Mars transponder link is not only feasible but quite robust. We also demonstrate through analysis the potential advantages of compact, low output power $(<300$ $\mathrm{mW}$ ), photon-counting transponders, which utilize NASA's developmental SLR2000 satellite laser ranging system as the Earth terminal and offer some concluding remarks regarding future applications. Published by Elsevier Science Ltd.
\end{abstract}

\section{Introduction}

Over 40 international stations, coordinated by the International Laser Ranging Service (ILRS), are dedicated to tracking approximately 25 Earth-orbiting artificial satellites around the clock in

E-mail address: jjd@1tpmail.gsfc.nasa.gov (J.J. Degnan). 
support of numerous Earth Science applications (Degnan, 1993). The satellites range in altitude from about 300 to 20,000 km (GPS, GLONASS, Etalon). Apollo 11 carried the first of five passive retroreflector arrays to the Moon in July 1969. Because of the great distance between the Earth and Moon ( $\sim 400,000 \mathrm{~km})$ and correspondingly weak signal returns, lunar laser ranging (LLR) has been routinely achieved by only three SLR stations over the past 32 years. To enhance the weak return signal, all successful LLR stations, including the NASA/GSFC funded stations at the McDonald Observatory in Texas and on Mt. Haleakala in Hawaii (now decommissioned) and the French CNES/CERGA Grasse Lunar Observatory, have been located at astronomical sites with above average atmospheric "seeing" in order to improve atmospheric transmission and preserve the narrow laser beam divergence. Furthermore, these stations originally employed the largest tracking telescopes and most powerful lasers in the international satellite laser ranging (SLR) network. In spite of these advantages, the mean received signal strength per laser fire is still well below a single photoelectron because of the familiar $\mathrm{R}^{-4}$ dependence of signal strength on range for a passive reflector (Degnan, 1993). In fact, the signal return rates from the Moon are sufficiently low that LLR can only be carried out at night and only during certain lunar phases. Near "Full Moon", the solar illumination of the lunar surface overwhelms the weak signal return from the target while, at "New Moon", no lunar surface features are visible to aid in guiding the extremely narrow laser beam to the target. The lunar systems also use special post-detection Poisson filtering techniques which extract the single photon range signal from background noise (Abbott et al., 1973).

In spite of the temporal gaps in lunar range data, the scientific information extracted from centimeter accuracy LLR data has been enormous. In addition to precise measurements of the lunar ephemeris and librations (deviations from uniform rotation) and of fundamental physical constants, lunar laser ranging provides insights into the internal makeup of the Moon and the Earth-Moon interaction and provides important tests of general relativity as well as constraints on its associated metrics (Dickey et al., 1994). The ability to extend these precise range measurements to the inner planets, or to spacecraft in orbit about the Sun, would certainly result in a similar array of important scientific results in the fields of planetary science, solar physics, fundamental physics, and general relativity.

Although modern SLR systems are capable of sub-cm ranging accuracies and 50 ps time transfer between remote clocks over typical near-Earth satellite distances (Degnan, 1993), extending these unique capabilities to the inner planets and beyond requires that we adopt a new approach. To overcome the prohibitively large $\mathrm{R}^{-4}$ signal loss characteristic of single-ended links to passive reflectors, we must utilize two-way laser links, which we will refer to generically as "transponders". In such systems, which have a transmitter and ranging receiver at both ends of the link, the signal strength at each terminal falls off only as $\mathrm{R}^{-2}$.

The use of microwave or laser transponders had previously been proposed for lunar ranging (Bender et al., 1990). Laser transponders on the Moon would make lunar ranging accessible to the smallest of the global SLR stations, and the resulting orders-of-magnitude higher signal strengths would remove the current tracking limitations near "New" and "Full" Moon. The NASA Goddard Space Flight Center is presently working with colleagues in Japan and Germany to propose a laser transponder on the Selene II Lunar Lander Mission (Schreiber et al., 1999), which has been suggested as a possible 2006 follow-on to the Japanese Selene lunar lander mission in 2003. 
To date, the most precise ranging to another planet was achieved when the radio telescopes of the NASA/JPL Deep Space Network (DSN) were in communication with the Viking Lander on Mars. This two-way microwave link typically resulted in several meter precision measurements, with 3 meters precision being the best internal consistency ever reported over a short tracking interval (Lemoine, private communication). The absolute accuracy of these interplanetary measurements, however, is limited by the presence of the interplanetary solar plasma, which, like the Earth's similarly charged ionosphere, can have a significant, and largely unknown, impact on the propagation delay at microwave frequencies. Optical frequencies, on the other hand, are much too high to be affected by the charged solar plasma. Furthermore, the shorter optical wavelengths characteristic of lasers allow the transmitted energy to be propagated in highly collimated (few arcsecond divergence) beams and therefore require much smaller collecting and transmitting apertures at both ends of the link. Of course, mutual high accuracy pointing between the space and ground terminals is required to take advantage of the narrow laser beam divergence. Furthermore, because of the long pulse transit times between terminals, each of the remote terminals must be able to independently detect, identify, and lock onto the signals from the opposite terminal using only a priori information stored at that terminal.

The feasibility of interplanetary laser transponders has been discussed by the author in the Proceedings of the last three International Workshops on Laser Ranging (Degnan, 1996, Degnan et al., 1998; Degnan 2000). The present paper seeks to review and amplify on some of this earlier material, provide detailed derivations of the various equations and models used, and provide a complete analysis of an Earth-Mars link. In Section 2 of this paper, we will describe two types of transponders (echo and asynchronous), introduce the transponder link equation, describe how range and time can be transferred between terminals, and preview the potential advantages of photon counting asynchronous transponders for interplanetary applications. In Section 3, we will provide mathematical models for the various sources of noise in an interplanetary transponder link including planetary albedo, solar or lunar illumination of the local atmosphere, and laser backscatter off the local atmosphere. Detailed derivations of the noise models can be found in Appendix A. In Section 4, we discuss the key components of an interplanetary laser transponder and develop an operational scenario for the acquisition and tracking of the opposite terminal. In Section 5, we use the theoretical models of the previous sections to perform two Earth-Mars link analyses over a full synodic period of 780 days. In the first example, we demonstrate that, using slightly modified versions of existing space and ground based laser instruments, an Earth-Mars transponder link is not only feasible but quite robust. In the second example, we describe the potential advantages of compact photon-counting transponders, which use NASA's developmental SLR2000 satellite laser ranging system as the Earth terminal. The latter system uses few $\mathrm{mm}$ long, passively Q-switched Nd:YAG microchip lasers to generate subnanosecond pulses (Degnan and Zayhowski, 1998). Section 6 provides a summary of the results and some concluding remarks regarding future applications.

We are primarily concerned with order of magnitude effects in this paper. Therefore, in generating our numerical results, we will assume that the Earth and Mars follow simple circular, concentric, coplanar orbits about the Sun. Results obtained from the use of more complicated state-of-the-art planetary ephemerides are quoted as needed to make a necessary point. Similarly, we do not consider the comparatively small relativistic effects that spacecraft velocity and local gravity may have on some of the quantities discussed here. 


\section{Basic transponder theory}

\subsection{Transponder types}

Transponders fall into two basic classes, i.e. echo and asynchronous. The type typically used in underwater acoustic sounders or microwave aircraft transponders is the echo transponder in which a pulse emitted from terminal $\mathrm{A}$ is detected by terminal $\mathrm{B}$ which then generates a response pulse subsequently detected by A. The delay between the received and transmitted pulse at the secondary terminal B is usually either known a priori through careful calibration or controlled via active electronics and then subtracted from the observed roundtrip time before computing the target range. Alternatively, the delay can be measured locally at terminal B and transmitted to the primary terminal A via a communications link. The signal return rate at the primary station is then equal to the fire rate of the laser multiplied by the joint probability that pulses are detected at both ends of the link. Thus, this simple echo approach works very well when the roundtrip time-of-flight is relatively short and there is a high probability of detection at both ends of the link, i.e. when both the uplink and downlink signal is reasonably strong and pointing uncertainties are small relative to the transmitter divergence. However, in situations where the light transit time is relatively long and the probability of detection is small at one or both ends of the link, it is worthwhile considering an alternative approach, i.e., the asynchronous laser transponder.

In an asynchronous transponder, the two terminals independently fire pulses at each other at a known laser fire rate, $f_{q s}$, as illustrated by the timing diagram in Fig. 1. For the purposes of this discussion, we will adopt the convention that Terminal A resides closest to the Sun and Terminal B is the outermost terminal. For an Earth-Mars link, for example, the Earth terminal records the times of departure of its own transmitted pulses $\left(t_{\mathrm{A} 1}\right)$ as well as the times of arrival of pulses from Mars $\left(t_{\mathrm{A} 2}\right)$ and vice versa. In a high SNR system, the pulses arrive at roughly the laser fire rate

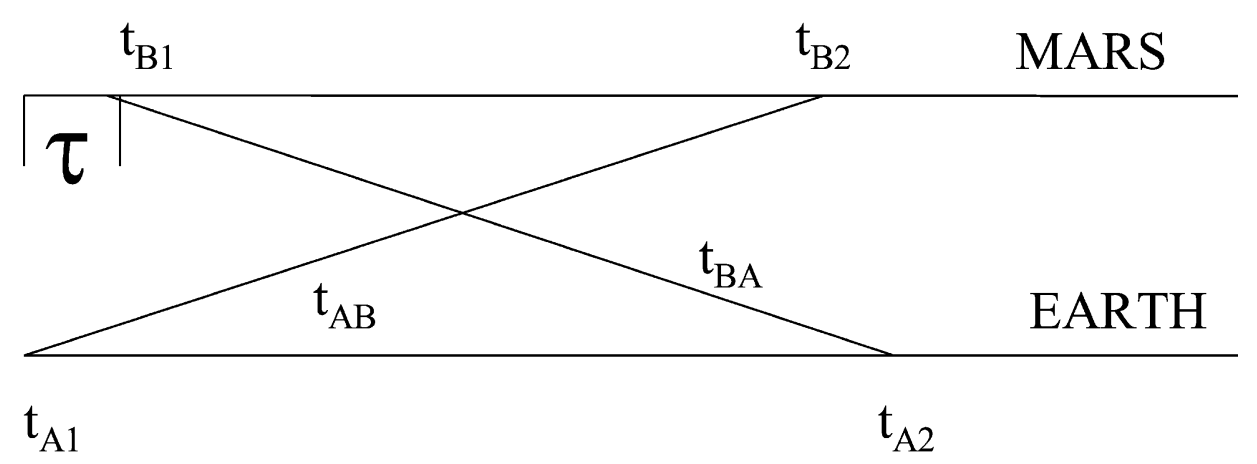

Fig. 1. Timing diagram for an asynchronous laser transponder on Mars exchanging pulses with an Earth station. A pulse leaves the Earth station at time $t_{\mathrm{A} 1}$, travels to Mars in a time $t_{\mathrm{AB}}$, and arrives at Mars at time $t_{\mathrm{B} 2}$. Similarly, a pulse is transmitted from Mars at time $t_{\mathrm{B} 1}$, travels to Earth in a time $t_{\mathrm{BA}}$, and arrives on Earth at time $t_{\mathrm{A} 2}$. Times of departure and arrival are referenced to that terminal's clock, and the Mars values are transmitted to Earth over a microwave or laser communications link. The quantity $\tau$ is the actual time interval between the departure of pulses from the Earth and Mars stations and, combined with the outgoing pulse times as recorded by their respective clocks, provides the offset between the ground and spacecraft clocks. 
whereas, in low SNR systems, the pulses may arrive intermittently. The departure and arrival times measured at each terminal are then communicated to, and properly paired at, an Earthbased processor which then calculates a range and clock offset between the two terminals for each set of two way measurements occurring within a reasonably short time interval. In a manner analogous to the familiar lock-in amplifier, approximate a priori knowledge of the laser fire rate at the opposite terminal, as well as the range-rate between terminals, allows the special correlation range receiver (CRR) to independently extract the relatively scarce, but temporally coherent, signal photons from the more prevalent, but randomly distributed, background noise photons (Degnan, 2002, this issue).

\subsection{Two-way measurement of range and clock offset}

With an asynchronous transponder, any pair of photon timing events originating from opposite terminals allow the post facto calculation of an instantaneous spacecraft range and spacecraft clock offset via the equations:

$$
R=\frac{c}{2}\left(t_{\mathrm{BA}}+t_{\mathrm{AB}}\right)=\frac{c}{2}\left[\left(t_{\mathrm{A} 2}-t_{\mathrm{A} 1}\right)+\left(t_{\mathrm{B} 2}-t_{\mathrm{B} 1}\right)\right]
$$

and

$$
\tau=\frac{\left[\left(t_{\mathrm{A} 2}-t_{\mathrm{A} 1}\right)-\left(t_{\mathrm{B} 2}-t_{\mathrm{B} 1}\right)\right]}{2\left(1+\frac{\dot{R}}{c}\right)}
$$

where the intervals $\left(t_{\mathrm{A} 2}-t_{\mathrm{A} 1}\right)$ and $\left(t_{\mathrm{B} 2}-t_{\mathrm{B} 1}\right)$ are measured by Terminals $\mathrm{A}$ and $\mathrm{B}$ respectively. In (1) and (2), $R$ and $\tau$ are the instantaneous range and clock offset at the point in time when the "photon world lines" marked $t_{\mathrm{AB}}$ and $t_{\mathrm{BA}}$ in Fig. 1 intersect as can be deduced by simple symmetry arguments. In (2), the small correction term, $R / \mathrm{c}$, corresponds to the instantaneous range rate between the Earth station and the spacecraft divided by the speed of light and can be estimated from a priori planetary ephemerides, the doppler shift in the microwave communications link, or iteratively solved for from the laser range data. Recent analyses for the Earth-Mars (Lemoine, private communication) and Earth-Vesta (Giorgini, private communication) links, using ephemerides provided by JPL, have both shown that, while the error in a priori interplanetary range may be as much as several tens of kilometers, the error in the calculated range rate is quite small, typically a few $\mathrm{mm} / \mathrm{sec}$. As will be discussed in Section 4.3, this small error in the computed range rate is crucial to reliable acquisition of weak signals in the presence of background noise.

\subsection{Accuracy and variance of the range and clock offset measurements}

Since pulse transit times between planets are on the order of several minutes, spacecraft clock frequency offsets and instabilities dominate the systematic and random interplanetary ranging errors respectively whereas, for conventional satellite laser ranging, the light roundtrip transit time is typically measured in milliseconds, and uncertainties in the atmospheric propagation delay 
dominate the systematic error at the sub-cm level. From Eqs. (1) and (2), the systematic errors in the measured range and clock offsets due to a constant frequency offset error in the Earth and/or spacecraft clocks from some nominal frequency $f_{c}$ are given by the equations

$$
\Delta R=\frac{c}{2}\left[\Delta\left(t_{\mathrm{A} 2}-t_{\mathrm{A} 1}\right)+\Delta\left(t_{\mathrm{B} 2}-t_{\mathrm{B} 1}\right)\right] \approx \frac{R}{2}\left[\frac{\Delta f_{\mathrm{A}}}{f_{c}}+\frac{\Delta f_{\mathrm{B}}}{f_{c}}\right]
$$

and

$$
\Delta \tau=\frac{\left[\Delta\left(t_{\mathrm{A} 2}-t_{\mathrm{A} 1}\right)-\Delta\left(t_{\mathrm{B} 2}-t_{\mathrm{B} 1}\right)\right]}{2\left(1+\frac{\dot{R}}{c}\right)} \approx \frac{R}{2 c\left(1+\frac{\dot{R}}{c}\right)}\left[\frac{\Delta f_{\mathrm{A}}}{f_{c}}-\frac{\Delta f_{\mathrm{B}}}{f_{c}}\right]
$$

We see from the latter equations that the fractional error in range is equal to the average of the fractional frequency offsets in the two clocks whereas the fractional error in the clock offset determination is proportional to the difference between the fractional frequency offsets in the two clocks. The variance in the range and offset measurements is in turn given by

$$
\left\langle\Delta R^{2}\right\rangle \approx R^{2}\left[\frac{\left\langle\Delta f_{\mathrm{A}}^{2}\right\rangle}{f_{c}^{2}}+\frac{\left\langle\Delta f_{\mathrm{B}}^{2}\right\rangle}{f_{c}^{2}}\right]
$$

and

$$
\left\langle\Delta \tau^{2}\right\rangle=\left[\frac{R}{2 c\left(1+\frac{\dot{R}}{c}\right)}\right]^{2}\left[\frac{\left\langle\Delta f_{\mathrm{A}}^{2}\right\rangle}{f_{c}^{2}}+\frac{\left\langle\Delta f_{\mathrm{B}}^{2}\right\rangle}{f_{c}^{2}}\right]
$$

for two independent free-running clocks.

Thus, the precision and accuracy of the single shot ranging and time transfer will be limited by the less accurate ground and spaceborne clocks. If the spaceborne clock had the stability of a good rubidium standard $\left(5 \times 10^{-13}\right)$, sub-decimeter level single shot range uncertainties would result for distances on the order of $1 \mathrm{AU}(\sim 150$ million $\mathrm{km})$. With respect to single shot time transfer, the latter clock would introduce a timing jitter on the order of $250 \mathrm{ps}(<4 \mathrm{~cm})$. However, if both clocks were of maser quality $\left(\sim 1 \times 10^{-15}\right.$ over time intervals of several minutes), clock errors would introduce submillimeter errors over distances on the order of $1 \mathrm{AU}$ and range accuracy would then be limited, as in conventional SLR to artifical satellites, at the sub-cm level by uncertainties in the atmospheric propagation paths.

One potential enhancement that has yet to be explored thoroughly is the degree to which an inferior spacecraft clock can be "disciplined" by an Earth-based maser during transponder operations via the laser time transfer process. This would be somewhat analogous to the present-day 
"disciplining" of quartz crystal and rubidium frequency references via frequent comparisons with the 1 pps output of GPS timing receivers. The latter receive timing signals from a maser-controlled satellite constellation and results in a long term frequency stabilization and performance of the slaved clock on a par with the master clock.

\subsection{Transponder link equation}

The mean number of signal photoelectrons recorded by the terminal B receiver on a single laser fire is given by the transponder link equation

$$
n_{s}^{B}=\frac{C_{\mathrm{AB}} E_{\mathrm{A}} A_{\mathrm{B}}}{R^{2}}
$$

where $E_{\mathrm{A}}$ is the pulse energy transmitted from terminal $\mathrm{A}, A_{\mathrm{B}}$ is the area of the receiving telescope at the receiving terminal $\mathrm{B}, \mathrm{R}$ is the distance between the two terminals, $C_{\mathrm{AB}}$ is a transponder constant given by

$$
C_{\mathrm{AB}}=\frac{\eta_{q}^{\mathrm{B}} \eta_{r}^{\mathrm{B}} T_{\mathrm{A}}^{\sec \left(\theta_{\mathrm{A}}\right)} T_{\mathrm{B}}^{\mathrm{sec}\left(\theta_{\mathrm{B}}\right)}}{h \nu \Omega_{t}^{\mathrm{A}}}
$$

$\eta_{q}^{\mathrm{B}}$ is the detector B quantum efficiency, $h v$ is the laser photon energy, $T_{\mathrm{A}}$ and $T_{\mathrm{B}}$ are the oneway zenith atmospheric transmissions at Terminals $\mathrm{A}$ and $\mathrm{B}$ (appropriate for two terminals on planetary surfaces), $\theta_{\mathbf{A}}$ and $\theta_{\mathbf{B}}$ are the local zenith angles for the opposite terminal, $\Omega_{t}^{\mathrm{A}}$ is the transmitter solid angle for laser $\mathrm{A}$, and $\eta_{r}^{\mathrm{B}}$ is the optical throughput efficiency of the receiver $\mathrm{B}$ optics respectively. The order of the subscripts in the transponder constant determines the direction of the link, i.e. $C_{\mathrm{AB}}$ is the link from terminal $\mathrm{A}$ to $\mathrm{B}$. The mean signal at the opposite terminal is obtained by simply interchanging $\mathrm{A}$ and $\mathrm{B}$ in the above equations.

\section{5. "Balanced” transponder links}

We can choose to write (7) in terms of the average laser power, i.e.

$$
n_{s}^{\mathrm{B}}=\frac{C_{\mathrm{AB}} P_{\mathrm{A}} A_{\mathrm{B}}}{f_{\mathrm{qs}} R^{2}}
$$

where $f_{\mathrm{qs}}$ is the Q-switching frequency (laser repetition rate) in $\mathrm{Hz}$ and we refer to $P_{\mathrm{A}} A_{\mathrm{B}}$ as the Mixed Power-Aperture Product to reflect the fact that it contains properties of two different terminals. Although not essential, the use of a common laser fire rate in the double-ended transponder link conceptually simplifies the instrument. From (9), the ratio of the mean signal strengths at the two terminals is given by

$$
\frac{n_{s}^{\mathrm{B}}}{n_{s}^{\mathrm{A}}}=\frac{C_{\mathrm{AB}}}{C_{\mathrm{BA}}} \frac{P_{\mathrm{A}} A_{\mathrm{B}}}{P_{\mathrm{B}} A_{\mathrm{A}}} \cong \frac{P_{\mathrm{A}}}{A_{\mathrm{A}}} \frac{A_{\mathrm{B}}}{P_{\mathrm{B}}}
$$


where, from (8), the approximation holds if the laser beam divergence and detector and optical throughput efficiencies are roughly equal at both ends of the link $\left(C_{\mathrm{AB}} \cong C_{\mathrm{BA}}\right)$. Furthermore, the above ratio is approximately unity if the power-to-aperture ratio is the same at both ends of the transponder link; i.e. $P_{\mathrm{A}} / A_{\mathrm{A}}=P_{\mathrm{B}} / A_{\mathrm{B}}$. We will refer to this as a balanced system. Note that the signal detection rate is proportional to the mixed power-aperture product, $P_{\mathrm{A}} A_{\mathrm{B}}$, and that, for a balanced system, the frequency of measurement at both terminals is approximately equal. Thus, if it becomes necessary to conserve limited spacecraft resources while still maintaining a balanced link with a fixed sampling rate, one can increase the power and telescope aperture at the Earth station proportionally to maintain the same Power-to- Aperture ratio while simultaneously reducing the transponder laser power and receive aperture at the remote terminal by the same factor.

\subsection{Maximizing the sampling rate through single photon detection}

The mean number of range returns per second recorded at either terminal is given by the product of the laser fire rate and the probability of detection for that terminal, i.e.

$$
f_{S}=f_{q s} P_{D}\left(n_{s}, n_{t}\right)=f_{\max }\left[\frac{1}{n_{s}}\left(1-e^{-n_{s}} \sum_{k=0}^{n_{t}-1} \frac{n_{s}^{k}}{k !}\right)\right]
$$

where we have solved for $f_{q s}$ in (9). The probability of detection in the low signal limit is given by Poisson statistics and depends on both the mean signal strength, $n_{s}$, and the detection threshold, $n_{t}$. Thus, for terminal $\mathrm{B}$, the maximum range sampling rate is

$$
f_{\max }^{\mathrm{B}}=\frac{C_{\mathrm{AB}} P_{\mathrm{A}} A_{\mathrm{B}}}{R^{2}}
$$

In Fig. 2, we plot the normalized detection rate, $f_{s} / f_{\max }$ and demonstrate that the parameter $f_{\max }$ corresponds to the maximum detection rate in the limit of small mean signal strength and single photoelectron detection. We draw the following conclusions from the figure:

For a given mixed power-aperture product, the maximum range sample rate is obtained by using single photon detection and a high repetition rate laser with low energy per pulse such that the mean signal count received per laser fire is $n_{s} \leqslant 0.1$.

For higher detection thresholds, the frequency of signal returns peaks at a value lower than $f_{\max }$ for mean signal counts near the threshold value (e.g. at $n_{s}=1.8$ pe for $n_{t}=2$ pe and at $n_{s}=3.3$ pe for $n_{t}=3$ pe, etc.). To achieve comparable return rates at thresholds higher than one photoelectron, the instrument power-aperture product would have to be increased significantly (e.g. by factors of 3.4 and 5.1 for thresholds of 2 and 3 pe respectively).

In addition to the sampling rate advantage, there is much to be gained from a size, weight, and power efficiency standpoint if one operates in single photon detection mode at both ends of the 


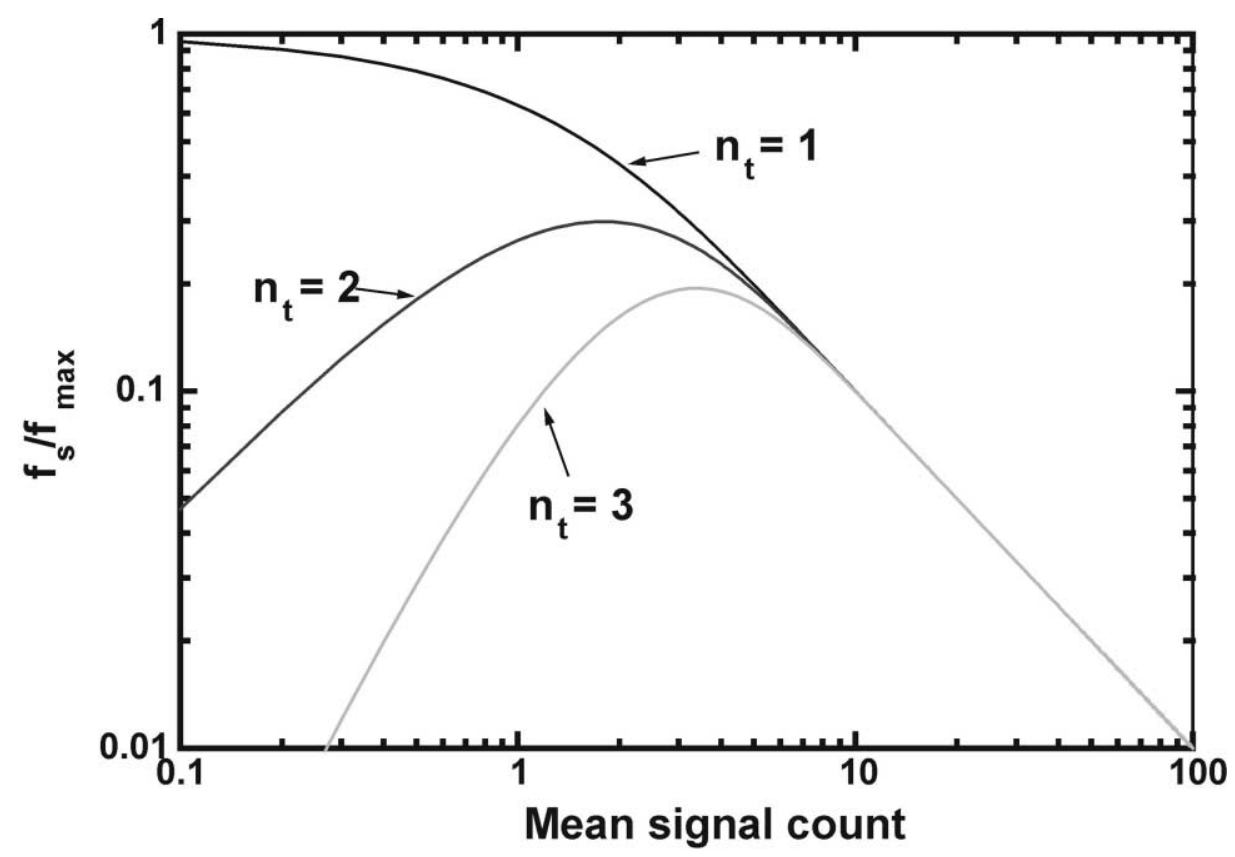

Fig. 2. Range sampling rate as a function of the detection threshold, $n_{\mathrm{t}}$, and the mean signal count, $n_{\mathrm{s}}$, normalized for a particular Mixed Power-Aperture Product and range.

link. This is especially relevant when one considers interplanetary distances of several hundred million $\mathrm{km}$ and the $R^{-2}$ dependence on signal strength. Severe size, weight, and prime power limitations on interplanetary spacecraft typically preclude either the use of large and powerful laser transmitters to enhance the signal strength at the Earth station or large telescopes to increase the signal strength at the spacecraft. Fortunately, as mentioned previously, one can place as much of the transponder link burden on the Earth station as necessary (within reasonable engineering or economic limits) in order to achieve a "balanced" and robust link. While Fig. 2 provides us with some interesting food for thought, a practical transponder link requires that each terminal have the ability to independently identify and extract single photon signals from the opposite terminal from the relevant noise background.

\section{Ranging receiver}

\subsection{Sources of noise}

Noise count rates can vary widely depending on whether the transponder is operating (1) in cruise phase between planets, (2) in orbit about the target planet, or (3) from the surface of a planet containing a scattering atmosphere. In this and subsequent sections, we will use the term "planet" in its broadest sense to include not only the major planets but also the minor planets (asteroids), moons of major planets, etc. 
The following three noise sources are present in all of the aforementioned operational scenarios: detector dark counts (internal noise unrelated to the local light environment), solar radiation scattered from the surface and atmosphere of the planet being viewed by the receiver (planetary albedo), and the residual stellar background in the receiver field of view (FOV). Direct solar illumination of the transponder optics and the resulting scatter within the instrument is another potential source of background noise. At Mars, for example, the Sun is never more than about $41^{\circ}$ away from the transponder line-of-sight to Earth. Stray light rejection is therefore an important consideration for any practical instrument when the opposite terminal is angularly close to the Sun and may dictate the use of light baffling in the telescope, internal light tubes, and an enclosed detector box to limit the amount of scattered light getting to the detector.

The number of counts from the star background within a narrow receiver FOV on the order of $100 \mu \mathrm{rad}$ (20 arcseconds) are expected to be relatively small compared to those induced by planetary reflections or emissions. Mars under maximum solar illumination, for example, appears as a magnitude -2.5 star when viewed from Earth (Zissis, 1993). Dark count rates in the visible detectors typically used in laser ranging also tend to be relatively low $\left(10^{2}-10^{4}\right.$ counts/s). Conventional Bi-Alkali or Multi-Alkali PhotoMultiplier Tubes (PMT's) typically have far lower dark count rates than Silicon-based Avalanche Photo-Diodes (APD's), and, when manufactured with more exotic cathodes such as GaAs or GaAsP, have demonstrated quantum efficiencies at $532 \mathrm{~nm}$ of 30 to $40 \%$.

An approximate expression for the noise count rate for the total planetary albedo (Planet A's surface and atmosphere), as viewed by the receiver at Terminal B, is derived in Appendix A1, i.e.

$$
\stackrel{\bullet}{n_{\mathrm{sa}}^{B}} \cong \frac{\eta_{q}^{B} \eta_{r}^{B}}{h v} \frac{N_{\lambda}^{A} \Delta \lambda_{\mathrm{B}} A_{\mathrm{B}} r_{\mathrm{A}}^{2} T_{\mathrm{B}}^{\mathrm{sec} \theta_{\mathrm{B}}}}{R^{2}}\left[\rho_{\mathrm{A}} g_{\mathrm{ps}}^{B}\left(\alpha_{A}\right)+\frac{1}{2} \ln \left(\frac{1}{T_{\mathrm{A}}}\right) g_{\mathrm{as}}\left(\alpha_{\mathrm{A}}\right)\right]
$$

where $N_{\lambda}^{\mathrm{A}}$ is the exoatmospheric solar spectral irradiance at Planet $\mathrm{A}$ at the operating wavelength, $\Delta \lambda_{\mathrm{B}}$ is the FWHM bandpass of Terminal B's spectral filter, $r_{\mathrm{A}}$ is the mean volumetric radius of planet $\mathrm{A}$, and $\rho_{\mathrm{A}}$ is the globally averaged surface reflectivity of Planet $\mathrm{A}$. The functions $g_{\mathrm{ps}}^{\mathrm{B}}$ and $g_{\text {as }}^{\mathrm{B}}$ are geometric factors for the surface and atmospheric contributions, which depend on the fraction of the planetary surface illuminated by the Sun. The geometric factors fall monotonically from a maximum value of 1 for full solar illumination to 0 for no illumination. The latter can be expressed as a function of the aspect angle, $\alpha_{A}$, defined as the angle subtended by two vectors, both originating from Planet A and directed toward the Sun and Planet B respectively as in Fig. 3.

For a planetary lander operating in local daylight, we must add the noise background rate caused by solar scattering in the local atmosphere. This is given by the following expression derived in Appendix A2,

$$
\begin{aligned}
\stackrel{\bullet}{n_{l s}^{\mathrm{B}}} & =\frac{\eta_{q}^{\mathrm{B}} \eta_{r}^{\mathrm{B}}}{h v} \frac{N_{\lambda}^{\mathrm{B}}\left(\Delta \lambda_{\mathrm{B}}\right) A_{\mathrm{B}} \Omega_{r}^{\mathrm{B}}}{4 \pi}\left\{\sec \theta_{\mathrm{B}} T_{\mathrm{B}}^{\mathrm{sec} \theta_{\mathrm{B}}}\left[\frac{1-T_{\mathrm{B}}^{\sec \theta_{\mathrm{s}}-\sec \theta_{\mathrm{B}}}}{\sec \theta_{\mathrm{S}}-\sec \theta_{\mathrm{B}}}\right]\right\} \\
& \simeq \frac{\eta_{q}^{\mathrm{B}} \eta_{r}^{\mathrm{B}}}{h v} \frac{N_{\lambda}^{\mathrm{B}}\left(\Delta \lambda_{\mathrm{B}}\right) A_{\mathrm{B}} \Omega_{r}^{\mathrm{B}}}{4 \pi}\left\{T_{\mathrm{B}}^{\sec \theta_{\mathrm{B}}} \ln T_{\mathrm{B}}^{\sec \theta_{\mathrm{B}}}\right\} .
\end{aligned}
$$

where $\Omega_{r}^{\mathrm{B}}$ is the receiver field of view in steradians. It should be mentioned that the atmospheric noise model makes no assumptions regarding the distribution of scatterers with altitude. The 

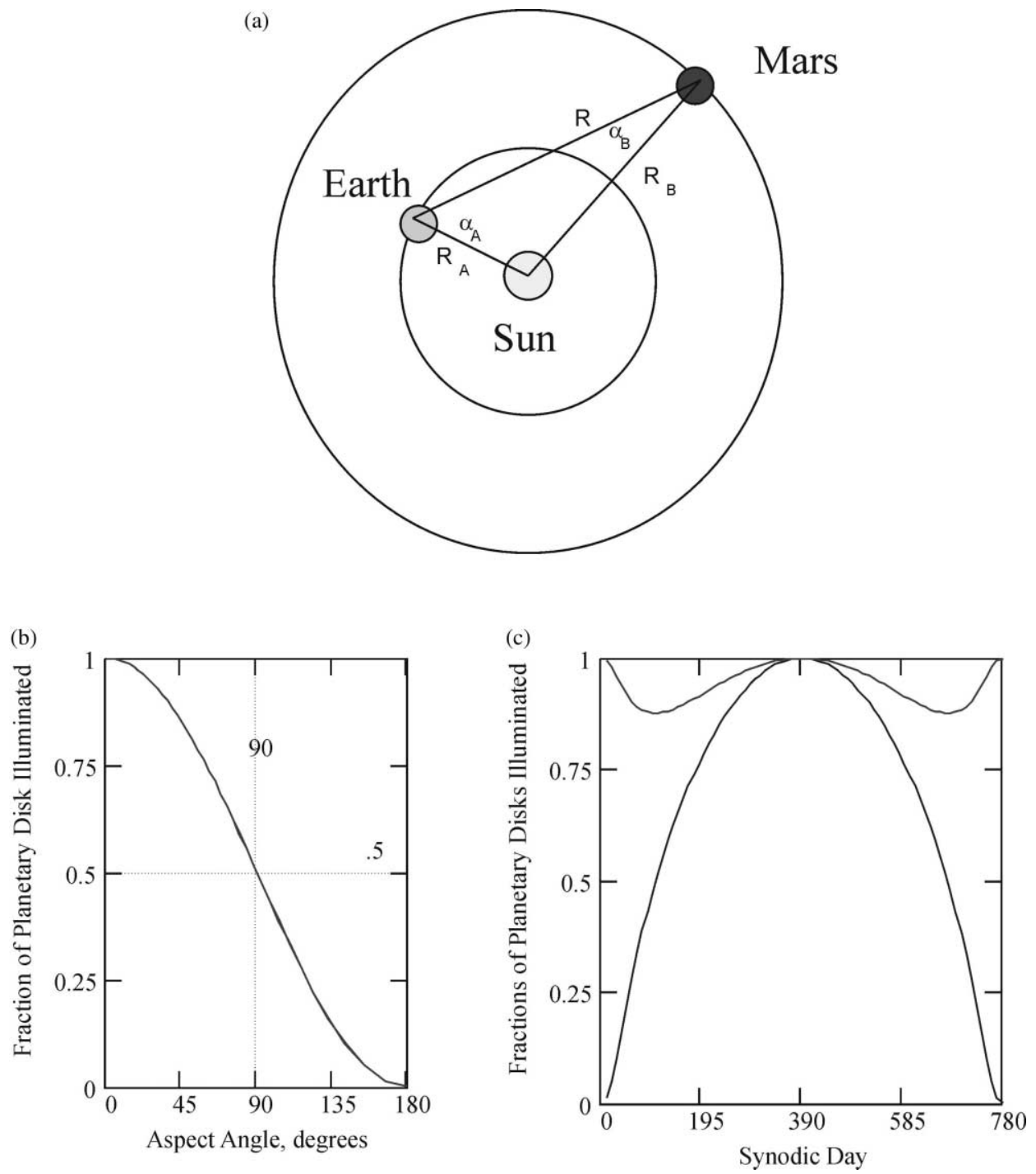

Fig. 3. (a) Solar system view of Planets A and B circling the Sun. The aspect angle for Planet A is defined as the angle formed by two vectors originating at Planet A and directed toward the other two bodies and vice versa. (b) The fraction of the planetary disk illuminated by the Sun as a function of aspect angle. (c) The fraction of the Earth (lower curve) and Mars (upper curve) disk illuminated by the Sun as viewed by the transponder terminals as a function of synodic phase (see Section 5). In our modeling of planetary albedo noise, each planet is assumed to be a solid sphere whose surface is characterized by a globally averaged surface reflectivity, $\rho$, with a thin atmospheric shell characterized by a zenith transmission, $T$. Planetary albedo noise is generated from any point on the planet where the solar illumination and receiver FOV overlap.

model makes the common assumption of no horizontal gradients, however, and therefore depends only on the zenith transmission, $T_{\mathrm{B}}$, between the station and the "top" of the planetary atmosphere and the local zenith angles of the Sun, $\theta_{S}$, and of the opposite terminal, $\theta_{\mathrm{B}}$. Note that as $T_{\mathrm{B}} \rightarrow 1$ (no scattering), the background count rate due to solar scatter off the atmosphere 
correctly goes to zero for all values of $\theta_{\mathrm{S}}$ and $\theta_{\mathrm{B}}$. The second expression in (14) is an approximation which is independent of $\theta_{\mathrm{S}}$ and $\theta_{\mathrm{B}}$. Although the approximation is strictly valid when $\theta_{\mathrm{S}} \sim \theta_{\mathbf{B}}$ or when $T_{\mathbf{B}}$ is close to unity, it also gives good results for $\theta_{\mathbf{B}}<60^{\circ}$ for nominally clear atmospheres $\left(T_{\mathrm{B}} \geqslant 0.7\right)$ and all values of $\theta_{\mathrm{S}}$ as demonstrated in Fig. 4.

During local night operations, the irradiance of the local atmosphere by nearby planetary moons produces background rates that are negligible when compared to planetary albedo. For example, a "full" Moon produces a noise background at the Earth terminal approximately six orders of magnitude less intense than the Sun. As a test, (13) was found to correctly predict the accepted spectral irradiance of the Full Moon as viewed from Earth, i.e $N_{\lambda}^{\mathrm{M}}=\rho_{\mathrm{A}} r_{\mathrm{A}}^{2} N_{\lambda}^{\mathrm{A}} / R^{2}=$ $4.8 \times 10^{-7} \mathrm{~W} / \mathrm{m}^{2}$-ster- $\mathrm{A}^{\circ}$. In this calculation, we used the exoatmospheric spectral radiance at Earth, $N_{\lambda}^{\mathrm{A}}=0.2 \mathrm{~W} / \mathrm{m}^{2}$-ster- $\mathrm{A}^{\circ}$, the mean volumetric radiance of the Moon, $r_{\mathrm{A}}=1738 \mathrm{~km}$, the mean Earth-Moon distance, $R=384,400 \mathrm{~km}$, and $\rho_{\mathrm{A}}=0.12$, a value in agreement with the known reflectivity of lunar soil in the visible (Zissis, 1993).

Back-scattered laser radiation produced by the outgoing pulse is an additional time-dependent source of noise and is applicable to both day and night operations from the planetary surface.

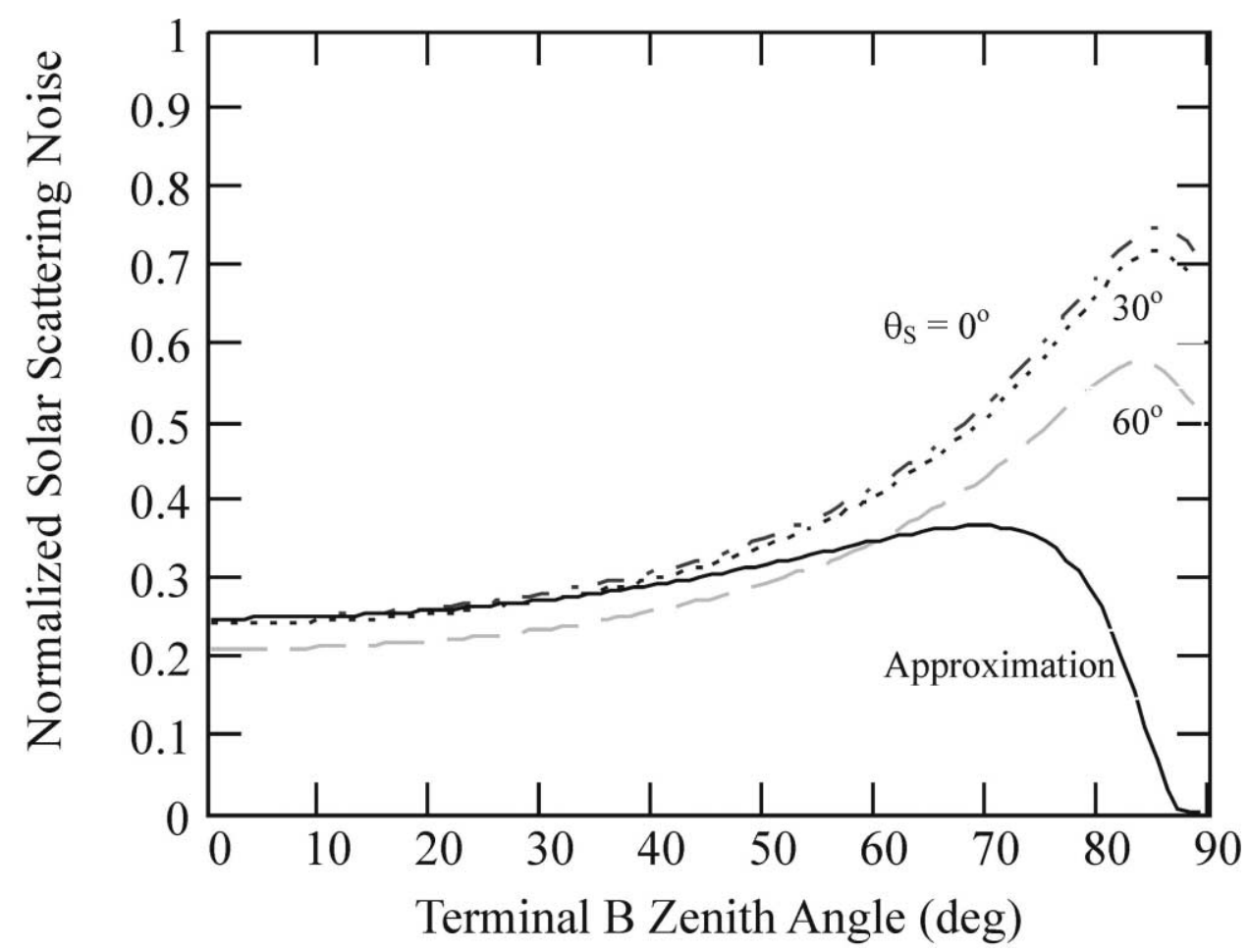

Fig. 4. Normalized contribution of local atmospheric scattering to the noise at Terminal A as a function of the Solar and Terminal $\mathrm{B}$ zenith angles, $\theta_{\mathrm{S}}\left(0^{\circ}, 30^{\circ}, 60^{\circ}\right)$ and $\theta_{\mathrm{B}}\left(0\right.$ to $\left.90^{\circ}\right)$, respectively as computed using (14) in the text. A standard clear Earth atmosphere (visibility $=23 \mathrm{~km}$ ) was assumed with a zenith transmission $T_{\mathrm{B}}=0.7$. The solid line represents the approximation in (14) which is independent of the solar zenith angle and valid under the assumption of high atmospheric transmission $\left(T_{\mathrm{B}} \sim 1\right)$ or $\theta_{\mathrm{S}} \sim \theta_{\mathrm{B}}$. The approximation is fairly good for $\theta_{\mathrm{B}}<60^{\circ}$ and improves for clearer or more transmissive atmospheres. 
For a monostatic system (coaxial transmitter and receiver optics), the latter contribution is given by the lidar equation (see Appendix A3)

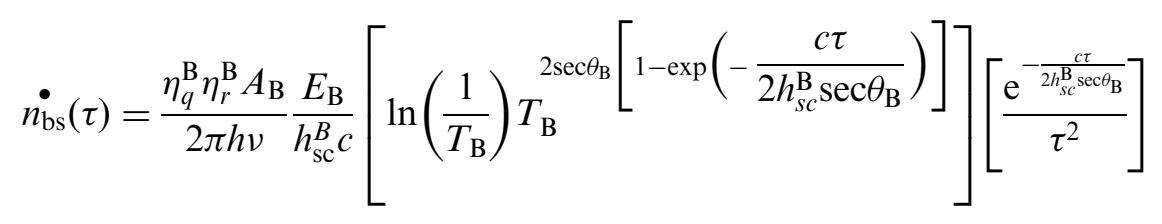

and falls off rapidly with the time from laser fire, i.e.

$$
\tau=\frac{2 s}{c}=\frac{2 \sec \theta_{\mathrm{B}}}{c}\left(z-h_{s}^{\mathrm{B}}\right),
$$

where $s$ is the distance from the terminal to the scattering volume, $h_{s}^{\mathrm{B}}$ is the altitude of the station from a nominal reference (e.g. sea level), $z$ is the corresponding altitude of the scattering volume, and $h_{s c}^{\mathrm{B}}$ is the atmospheric scale height. Due to the logarithmic term in (15a), the backscatter contribution correctly goes to zero in the absence of a scattering atmosphere. Laser backscatter can be greatly reduced through the use of bistatic optical systems (separate and non-coaxial transmit and receive optics).

\subsection{Correlation range receiver}

Every range receiver is characterized by a range gate, a range bin, and a detection threshold. In a single-ended range measurement to a passive reflector, the range gate, $\tau_{g}$, provides a temporal filter for the reduction of background noise and is chosen large enough to encompass the uncertainty in our a priori knowledge of the range to the target. The range bin, $\tau_{b}$, is the time over which the receiver integrates the incoming signal and is optimally chosen, in conventional high SNR systems, to be just large enough to capture the majority of the signal photons within a single pulse. In the case of photon-counting receivers (Degnan, 2002), a somewhat larger range bin is chosen to collect the photons from a collection of multiple pulses over some time interval referred to as a frame. In either case, the number of range bins within the range gate is given by the simple formula

$$
N_{\text {bin }}=\frac{\tau_{g}}{\tau_{b}}
$$

We refer to the 2D areas defined by the horizontal borders of the "range bins" and the vertical borders of the "frames" in Fig. 5 as cells. A consecutive sequence of frames is a super-frame (Degnan, 2002). We tentatively identify potential signal cells within a given frame by counting all of the photoelectrons generated within each cell and comparing it to a frame threshold. We refer to this basic data processing scheme, which was used successfully in LLR data processing (Abbott et al., 1973), as Post-Detection Poisson Filtering.

The strength of a ranging or communications link is often expressed in terms of a Signal-toNoise Ratio $(S N R)$. A closely related parameter, useful for photon counting receivers, is the signal cell contrast (Degnan, 2002). A third useful quantity for establishing the minimum laser pulse 


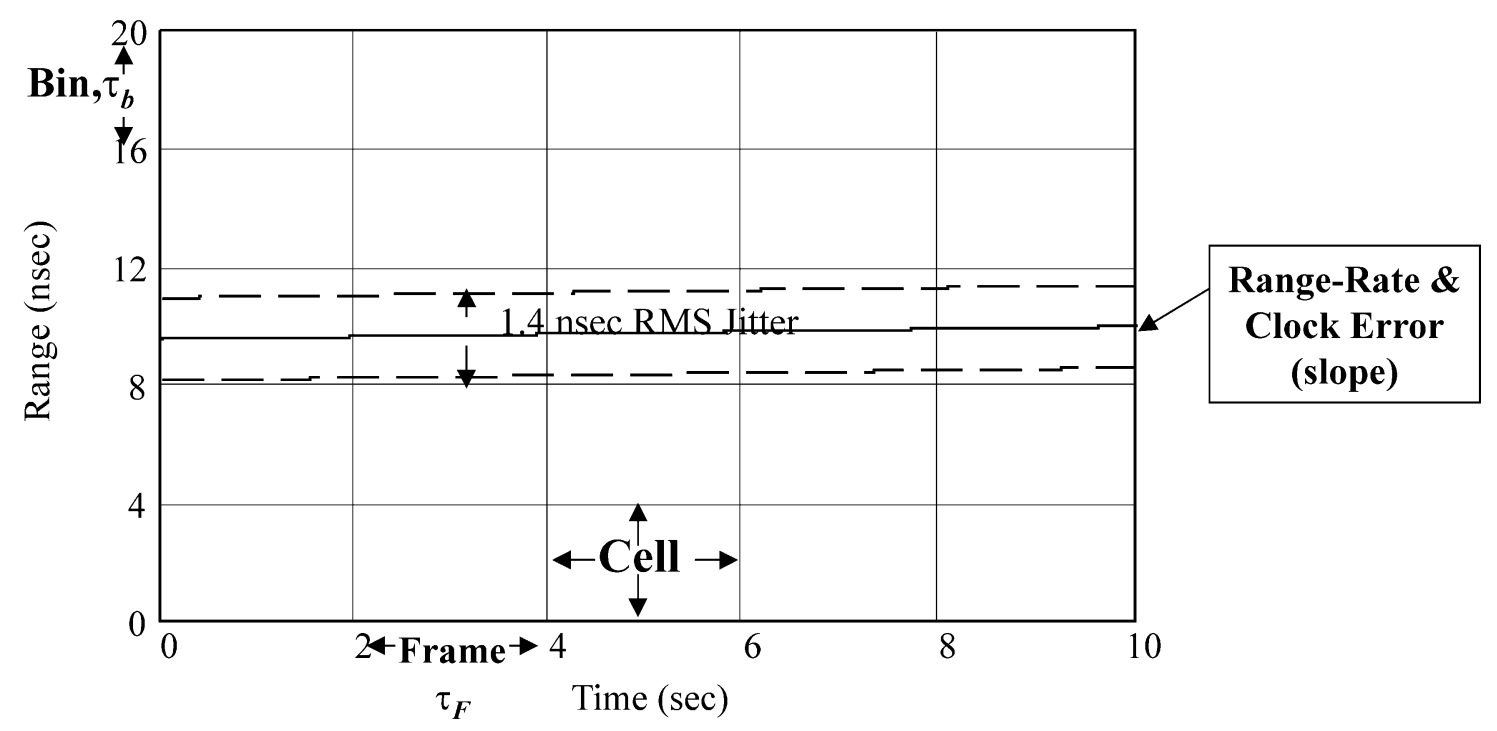

Fig. 5. The arrival times of incoming photons are recorded relative to when they are expected in an Observed Minus Calculated $(O-C)$ plot. In this reference system, a Correlation Range Receiver (CRR) divides the range gate in the vertical axis into range bins and the sequence of laser fires along the horizontal axis into frames. The bin and frame sizes are chosen to ensure high probability that the observed signal counts all fall within a single cell, i.e. the 2-D areas defined by the vertical borders of the frame and the horizontal borders of the bins. The choice of bin and frame size are intertwined and must take into account the basic precision of the measurement, which sets a minimum bin size, as well as the time it takes to collect enough signal counts to adequately discriminate the signal cell from the noise cells. Various residual uncertainties in the range rate, due to ephemerides and planetary rotation model errors, as well as clock frequency offsets, which can mimic a range-rate error via the Doppler equation, can cause the signal photons to fall along a sloped line with the random scatter, caused by laser fire or receiver timing jitter, superimposed.

energy needed to establish a robust transponder link is the scale energy (see Section 3.3). For Terminal $\mathrm{B}$, the three quantities are related by the expression

$$
C_{\mathrm{B}}=1+\frac{N_{\mathrm{S}}}{N_{b}}=1+\frac{N_{f} n_{s}}{N_{f} n_{b}}=1+\frac{n_{s}^{\mathrm{B}}}{n_{b}^{\mathrm{B}}} \equiv 1+S N R=1+\frac{E_{\mathrm{A}}}{E_{s c}^{\mathrm{A}}}
$$

where $N_{s}$ and $N_{b}$ are the mean number of signal and noise photons collected in the signal cell over $N_{f}$ consecutive laser fires respectively, $n_{s}^{\mathrm{B}}$ and $n_{b}^{\mathrm{B}}$ are the mean number of received signal and noise photoelectrons per range bin generated in a single laser fire, $E_{\mathrm{A}}$ is the energy transmitted by Terminal A, and $E_{s c}^{\mathrm{A}}$ is the scale energy for Terminal A. A transmitter with an output energy equal to the scale energy produces a $S N R=1$ or a contrast, $C_{\mathrm{B}}=2$.

For single pulse detection in a high SNR system, we have $N_{f}=1$, and the threshold is usually set by hardware. For low SNR photon-counting systems, the threshold is usually set by a combination of hardware (e.g. a range receiver or multichannel scalar) and software which compares the counts in each bin/cell to a frame threshold, $K$, and rejects counts below the threshold as probable noise (Degnan, 2002). In either case, choosing too high a threshold results in the loss of valid range returns, whereas choosing too low a threshold results in increased noise-induced false alarms. Furthermore, because of the potentially large number of cells in a frame (especially 
during acquisition when range uncertainties are largest), it is possible that a sizable number of noise cells in a given frame will be falsely identified as signal even when the probability of false alarm for any given noise cell is relatively small. One approach to optimizing the frame threshold is to maximize the Differential Cell Count (Degnan, 2002), defined as the mean number of correctly identified signal cells minus the mean number of false alarms in cells containing only solar background counts within a frame, i.e.

$$
\delta N=P_{d}^{\mathrm{B}}-N_{\mathrm{bin}} P_{f a}^{\mathrm{B}}
$$

where $P_{d}^{\mathrm{B}}$ is the probability of correctly identifying the signal cell within a frame and $P_{f a}^{\mathrm{B}}$ is the probability of falsely identifying a noise cell as signal. This approach yields an "optimum" threshold condition for Terminal B given by (Degnan, 2002)

$$
K_{\mathrm{opt}}^{\mathrm{B}}=\frac{N_{s}^{\mathrm{B}}+\ln \left(N_{\mathrm{bin}}\right)}{\ln C_{\mathrm{B}}}=\frac{\left(C_{\mathrm{B}}-1\right) N_{b}^{\mathrm{B}}+\ln \left(N_{\mathrm{bin}}\right)}{\ln C_{\mathrm{B}}}
$$

where $N_{\text {bin }}$ is the number of range bins defined by (16), $N_{s}^{\mathrm{B}}$ is the mean signal count in the signal cell, $N_{b}^{\mathrm{B}}$ is the mean noise count in any given cell, and $C_{\mathrm{B}}$ is the signal cell contrast defined by (17). From Poisson statistics, the probability of correctly detecting the signal cell is given by

$$
\begin{aligned}
P_{d}^{\mathrm{B}} & =\mathrm{e}^{-C_{B} N_{b}^{\mathrm{B}}} \sum_{k=K_{\mathrm{opt}}}^{\infty} \frac{\left(C_{\mathrm{B}} N_{b}^{\mathrm{B}}\right)^{k}}{k !} \simeq \frac{1}{\sqrt{2 \pi C_{\mathrm{B}} N_{b}^{\mathrm{B}}}} \int_{K_{\mathrm{opt}}}^{\infty} \mathrm{d} N \exp \left[-\frac{\left(N-C_{\mathrm{B}} N_{b}^{\mathrm{B}}\right)^{2}}{2 C_{\mathrm{B}} N_{b}^{\mathrm{B}}}\right] \\
& =\frac{1}{2}\left[1+\operatorname{erf}\left(\frac{C_{\mathrm{B}} N_{b}^{\mathrm{B}}-K_{\mathrm{opt}}^{\mathrm{B}}}{\sqrt{2 C_{\mathrm{B}} N_{b}^{\mathrm{B}}}}\right)\right]
\end{aligned}
$$

where, in the limit of "large" mean counts $(>15)$, one can use the Central Limit Theorem to approximate the Poisson distribution by a Gaussian (normal) distribution and $\operatorname{erf}(x)$ is the familiar error function. Similarly, the probability of false alarm is given by

$$
\begin{aligned}
P_{f a}^{\mathrm{B}} & =\mathrm{e}^{-N_{b}^{\mathrm{B}}} \sum_{k=K_{\mathrm{opt}}}^{\infty} \frac{\left(N_{b}^{\mathrm{B}}\right)^{k}}{k !} \simeq \frac{1}{\sqrt{2 \pi N_{b}^{\mathrm{B}}}} \int_{K_{\mathrm{opt}}}^{\infty} \mathrm{d} N \exp \left[-\frac{\left(N-N_{b}^{\mathrm{B}}\right)^{2}}{2 N_{b}^{\mathrm{B}}}\right] \\
& =\frac{1}{2}\left[1-\operatorname{erf}\left(\frac{K_{\mathrm{opt}}^{\mathrm{B}}-N_{b}^{\mathrm{B}}}{\sqrt{2 N_{b}^{\mathrm{B}}}}\right)\right]
\end{aligned}
$$

The error function expressions can be used in the usual case where the optimum threshold lies between the peaks of the noise and signal cell populations, i.e. if we satisfy the condition $N_{b}^{\mathrm{B}}<K_{\mathrm{opt}}<C_{\mathrm{B}} N_{b}^{\mathrm{B}}=N_{s}^{\mathrm{B}}+N_{b}^{\mathrm{B}}$. 
For weaker signals, a powerful second test can be applied, if necessary, by requiring that cells in adjoining frames be correlated. In the most general sense, this simply means that cells tentatively identified as containing "signal" in adjacent frames must obey applicable physical laws or constraints. For example, the physical laws governing planetary or spacecraft motion do not allow the transponder position to make unexpected discontinuous jumps into widely separated range bins between frames. Thus, we can define a "valid trajectory" as one where the planet or spacecraft position changes by no more than one range bin in moving between frames and moves monotonically and quasi-linearly in the correct direction on either side of the range extrema. This correlation requirement allows us to apply an " $\mathrm{N}$ of $\mathrm{M}$ " test on multiple cells which survive the initial threshold test within a superframe and consisting of $\mathrm{M}$ successive frames, and recover missing signal cells which might have failed to reach threshold due to lapses in instrument pointing, changes in the atmospheric transmission, etc. For signal verification, the " $\mathrm{N}$ of $\mathrm{M}$ " test requires that at least $\mathrm{N}$ cells, all satisfying the threshold criteria and lying on a valid trajectory, be detected within the $\mathrm{M}$ successive frames comprising the superframe. Based on successful application of this test, any signal data in the up to (M-N) signal cells, which may have originally failed to meet the threshold criteria, can be successfully restored via interpolation between frames (Titterton et al., 1998; Degnan, 2002).

\subsection{Computation of the scale energy for an Earth-Mars link}

In photon counting systems, it is usually possible to create a robust link using pulse energies on the order of the scale energy or greater. As the SNR or contrast increases, fewer pulses must be averaged within a cell to detect the signal with high confidence. Using (7) and (8) in (16), we can derive the following expression for the scale energy

$$
E_{\mathrm{sc}}^{\mathrm{A}}=\frac{n_{b}^{\mathrm{B}} R^{2}}{C_{\mathrm{AB}} A_{\mathrm{B}}}=\frac{h \nu \Omega_{t}^{\mathrm{A}} R^{2} \tau_{b}}{\eta_{q}^{\mathrm{B}} \eta_{r}^{\mathrm{B}} T_{\mathrm{A}}^{\mathrm{sec} \theta_{\mathrm{A}}} T_{\mathrm{B}}^{\mathrm{sec} \theta_{\mathrm{B}}} A_{\mathrm{B}}} \sum_{k} n_{k}^{\mathrm{B}}=\sum_{k} E_{k}^{\mathrm{A}}
$$

where the sum is over the relevant noise count rates described in Section 3.1. We also note from (22) that there is a scale energy, $E_{k}^{\mathrm{A}}$, for each noise source, and they are additive.

In the limiting case of single pulse detection where $\tau_{b}$ is approximately equal to the full laser pulsewidth, the latter equation yields a scale peak power for transmitter A,

$$
P_{\mathrm{sc}}^{\mathrm{A}}=\frac{E_{\mathrm{sc}}^{\mathrm{A}}}{\tau_{b}}
$$

Let us first consider a transponder that is either enroute to or in orbit about a target planet or is on the surface of a planet or other body having no atmosphere. The scale energy for the Earth terminal (A) is then dominated by planetary albedo, which the outgoing laser signal from Earth must overcome, plus any instrumentation noise, such as detector dark counts. From (13), the scale energy for solar scattering from the planetary surface and atmosphere (albedo) is given by

$$
E_{\mathrm{sa}}^{\mathrm{A}} \cong \frac{N_{\lambda}^{\mathrm{A}} \Delta \lambda_{\mathrm{B}} \Omega_{t}^{\mathrm{A}} r_{\mathrm{A}}^{2} \tau_{b}}{T_{\mathrm{A}}^{\mathrm{sec} \theta_{\mathrm{A}}}}\left[\rho_{\mathrm{A}} g_{\mathrm{ps}}^{\mathrm{B}}\left(\alpha_{\mathrm{A}}\right)+\frac{1}{2} \ln \left(\frac{1}{T_{\mathrm{A}}}\right) g_{\mathrm{as}}^{\mathrm{B}}\left(\alpha_{\mathrm{A}}\right)\right],
$$


which yields the Transmitter A pulse energy that produces the same number of signal counts in the signal cell as the albedo noise.

Similarly, we can use (14) and (22) to compute the scale energy for local atmospheric scattering of solar (or lunar) radiation,

$$
E_{l s}^{\mathrm{A}}=\frac{N_{\lambda}^{\mathrm{B}} \Delta \lambda_{\mathrm{B}} \Omega_{t}^{\mathrm{A}} \Omega_{r}^{\mathrm{B}} R^{2} \tau_{b}}{4 \pi} \frac{\sec \theta_{\mathrm{B}}}{T_{\mathrm{A}}^{\sec \theta_{\mathrm{A}}}}\left[\frac{1-T_{\mathrm{B}}^{\sec \theta_{S}-\sec \theta_{\mathrm{B}}}}{\sec \theta_{S}-\sec \theta_{\mathrm{B}}}\right] \cong \frac{N_{\lambda}^{\mathrm{B}} \Delta \lambda_{\mathrm{B}} \Omega_{t}^{\mathrm{A}} \Omega_{r}^{\mathrm{B}} R^{2} \tau_{b}}{4 \pi T_{\mathrm{A}}^{\sec \theta_{\mathrm{A}}} T_{\mathrm{B}}^{\sec \theta_{\mathrm{B}}}}
$$

In this instance, the source of the exoatmospheric spectral irradiance, $N_{\lambda}^{\mathrm{B}}$, is the Sun during local daylight transponder operations or possibly one or more moons during local night operations. Under most practical situations, the noise contribution of lunar scattering from the atmosphere tends to be negligible when compared to the planetary albedo.

Finally, we can compute the time-dependent scale energy for laser backscatter in the local atmosphere from (15) and (22), i.e.

$$
E_{\mathrm{bs}}^{\mathrm{A}}(\tau)=\frac{E_{\mathrm{B}} \Omega_{t}^{\mathrm{A}} R^{2} \tau_{b}}{2 \pi h_{\mathrm{sc}}^{\mathrm{B}} c \tau^{2}} e^{-\frac{c \tau}{2 h_{\mathrm{sc}}^{\mathrm{B}} \sec \theta_{\mathrm{B}}}} \ln \left(\frac{1}{T_{\mathrm{B}}}\right) T_{\mathrm{B}}^{\sec \theta_{\mathrm{B}}-\sec \theta_{\mathrm{A}}} T_{\mathrm{B}}^{-2 \sec \theta_{\mathrm{B}} \exp \left(-\frac{c \tau}{2 h_{\mathrm{sc}}^{b} \sec \theta_{b}}\right)}
$$

For Terminal B, the corresponding scale energies are obtained by simply interchanging A and B in the above equations.

Since all of the scale energies in (24) to (26) depend linearly on the transmitter solid angle, a low divergence beam clearly reduces the energy requirements on the link. From (24) and (25), noise reduction is an important second step since the scale energies for planetary albedo and local solar scattering both depend linearly on the spectral width of the bandpass filter, $\Delta \lambda$, and the receiver field-of-view (FOV), $\Omega_{r}$ (spectral and spatial filtering). Since all of the scale energies depend linearly on the range bin, $\tau_{b}$, it is necessary to apply all known a priori corrections to the raw range data to increase the "temporal coherence" of the signal (Degnan, 2001) in order to minimize the required transmit energy (temporal filtering). Finally, one must choose the detection threshold in an optimum manner (amplitude filtering) as given by (19).

We can now use (24) and (25) to compute the scale energies for the planetary albedo and solar scattering contributions to the noise as a function of synodic phase. The results are plotted in Fig. 6 for the Earth transmitter (blue) and the Mars transmitter (red). In computing the scale energies, we have used the planetary transmissions in Table 1, the instrument parameters listed in Tables 2 and 3, and nominal terminal zenith angles of $30^{\circ}$. We assume a range bin of $1 \mathrm{nsec}$ (single subnanosecond pulse detection). The scale energy scales proportionally with the range bin width. In all cases, the scale energy for both terminals (the solid curves in Fig. 6) is always much less than $100 \mu \mathrm{J}$, implying sub-mJ energies should be adequate for an Earth-Mars transponder link. Not surprisingly, the scale energy for the Mars transmitter is completely dominated by the need to overcome heavy solar scattering in the Earth's atmosphere. For the Earth transmitter, Earth albedo dominates local solar scattering in the Martian atmosphere except near the middle of the synodic cycle when the Earth is at its greatest distance from Mars and the contribution of Earth albedo to the overall noise background is therefore substantially reduced. 


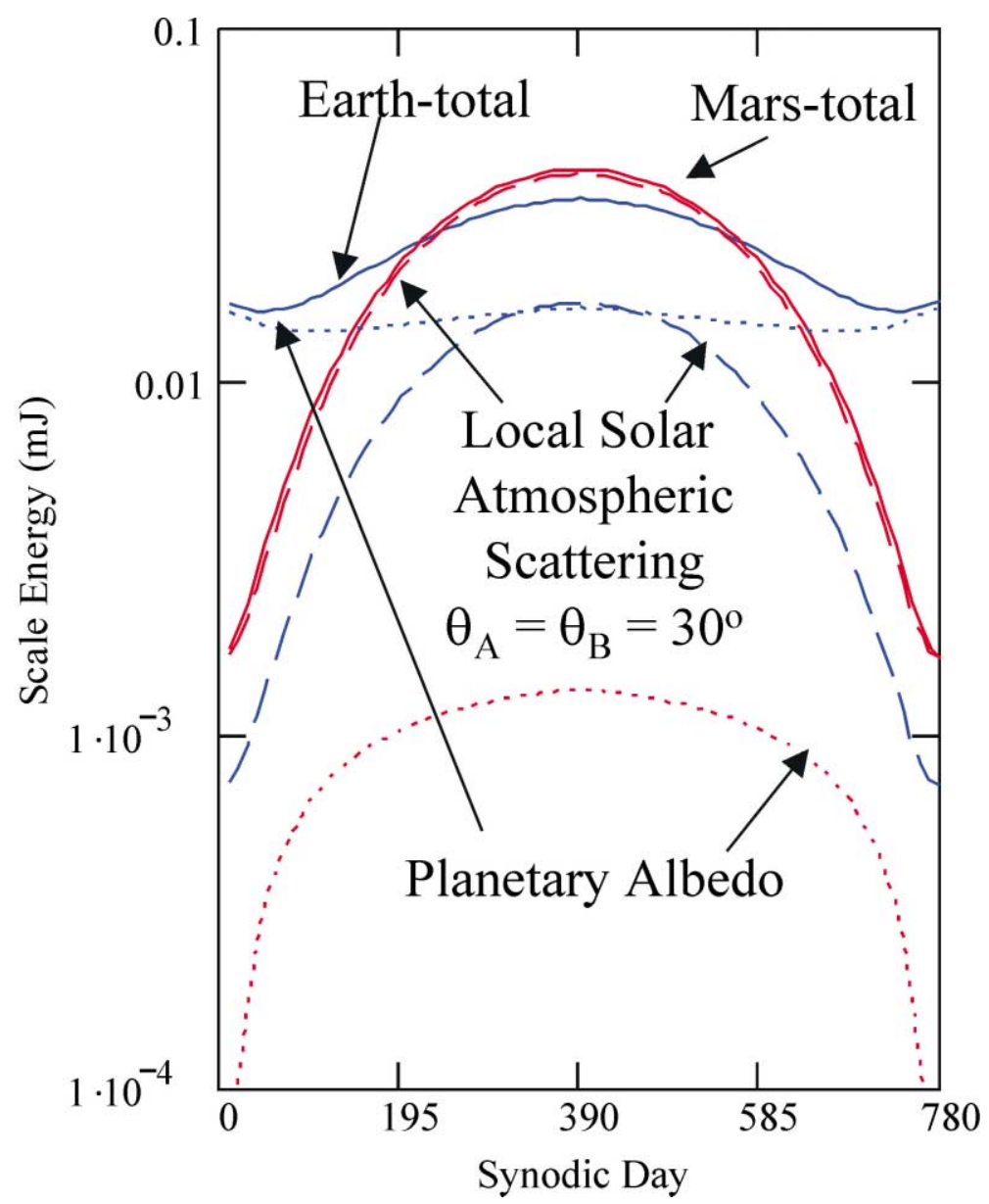

Fig. 6. Scale energies in microjoules as a function of synodic phase for both day and night operations with both terminals at a zenith angle of $30^{\circ}$. Scale energies for the Earth and Mars transmitters are represented by the blue and red curves respectively. The dotted lines represents the planetary albedo contribution and represents night operations. The dashed line represents the additional contribution from solar scattering in the local atmosphere. The solid line represents the sum of both scale energies representing local daylight operations.

\section{Acquisition of the Earth terminal}

A block diagram of a dual mode Microlaser Altimeter and Transponder (MAT) is provided in Fig. 7. When in orbit about the planet, the instrument ranges to the surface and provides topographic maps (Degnan, 2002). The same instrument can function as a transponder at any point within the mission, i.e. during interplanetary cruise phase, in orbit, or on the surface of the planet. In transponder mode, acquisition of the Earth terminal requires an initial search within a threedimensional volume bounded by the initial angular pointing uncertainty and the uncertainty in the time of arrival of the pulse from the opposite terminal. As mentioned previously, the latter determines the width of the range gate and has two components - the uncertainty in the a priori knowledge of range between the two terminals as derived from ephemerides and the uncertainty 
Table 1

Planetary parameters assumed in the link calculations

\begin{tabular}{lccc}
\hline Planetary parameter & Earth $(\mathrm{A})$ & Mars $(\mathrm{B})$ & Moon $\left(\mathrm{A}^{\prime}\right)$ \\
\hline Mean distance from Sun, R (AU $\left.\sim 150 \times 10^{6} \mathrm{~km}\right)$ & 1.0 & 1.52 & 1.0 \\
Orbital eccentricity & 0.0167 & 0.0935 & NA \\
Length of year, $\tau$ (Earth days) & 365.256 & 686.98 & NA \\
Length of day, h & 24 & 24.657 & NA \\
Obliquity of spin axis to orbital plane, deg & 23.45 & 25.19 & NA \\
Mean volumetric radius, km & 6371 & 3390 & 1738 \\
Mean surface reflectivity @ 532 nm, $\rho$ & 0.15 (est.) & 0.15 (est) & 0.12 \\
Zenith atmospheric transmission @ $532 \mathrm{~nm}, \mathrm{~T}$ & 0.7 & 0.9 (est) & 1.0 \\
\hline
\end{tabular}

Table 2

Instrumental parameters assumed in the link calculations for a conventional high SNR balanced transponder pair operating between Earth and Mars

\begin{tabular}{lcc}
\hline Parameter & MOBLAS (A) & "Green MOLA" (B) \\
\hline Transmitted pulse energy, mJ & 100 & 43 \\
Repetition rate, Hz & 5 & 5 \\
Average laser power, mW & 500 & 215 \\
FWHM pulsewidth, ps & 150 & $<1000$ \\
FWHM beam divergence, $\mu$ rad & 50 & 50 \\
Telescope diameter, cm & 76 & 50 \\
Detector quantum efficiency, $\%$ & $12 \%$ & $12 \%$ \\
Receiver optical throughput, \% & 40 & 40 \\
Receiver FOV, $\mu$ rad & 100 & 100 \\
Spectral bandwidth, nm & 0.3 & 0.3 \\
Range gate, ms (ungated) & 200 & 200 \\
Range bin, ns & 1 & 1 \\
Spacecraft Zenith angle, deg & 30 & 30 \\
\hline
\end{tabular}

in the laser fire time at the opposite terminal. As will be described in the following subsections, acquisition of the opposite terminal is most easily accomplished in two steps-first in 2-D angular space and then in 1-D range space.

\subsection{Initial acquisition of the earth station in angular space}

The angular search for the Earth terminal is aided by a sensitive CCD array capable of imaging the Earth, Moon, and nearby stars within a nominal $1^{\circ} \times 1^{\circ}$ field of angular uncertainty as in Fig. 8a. For a transponder mounted to the spacecraft body, this level of angular uncertainty is representative of the error associated with the pointing of a spacecraft from orbit or during interplanetary cruise phase. It is also inclusive of the expected angular error in the case where the transponder is assumed mounted to a meter-class K-band microwave communications dish communicating with Earth. Using pointing corrections to an independent two-axis transponder 
Table 3

Instrumental parameters assumed in the link calculations for a balanced, low SNR (photon-counting) transponder pair operating between Earth and Mars

\begin{tabular}{lcc}
\hline Parameter & SLR2000 (A) & "Green MLA" (B) \\
\hline Transmitted pulse energy, mJ & 0.130 & 0.053 \\
Repetition rate, Hz & 2000 & 2000 \\
Average laser power, mW & 260 & 103 \\
FWHM pulsewidth, ps & 200 & $<1000$ \\
FWHM beam divergence, $\mu \mathrm{rad}$ & 50 & 50 \\
Telescope diameter, cm & 40 & 25 \\
Detector quantum efficiency, $\%$ & $12 \%$ & $12 \%$ \\
Receiver optical throughput, \% & 40 & 40 \\
Receiver FOV, $\mu$ rad & 100 & 100 \\
Spectral bandwidth, nm & 0.3 & 0.3 \\
Range gate, ms (ungated) & 0.5 & 0.5 \\
Range bin, ns & 2 & 2 \\
Spacecraft Zenith angle, deg & 30 & 30 \\
\hline
\end{tabular}

gimbal mount of limited angular range $\left(<2^{\circ}\right)$, or alternatively a pair of Image Motion Compensators (IMC's) (McElroy et al., 1977), to implement the fine pointing of the receiver, the system computer can center and hold the Earth image in the CCD array. Space-qualified, high sensitivity CCD cameras with up to $2048 \times 2048$ pixel resolution are readily available and yield a $8.8 \mu \mathrm{rad}$ single pixel resolution for a nominal $1^{\circ} \times 1^{\circ}$ array FOV. Since the full Earth disk subtends an angular width between 34 and $163 \mu \mathrm{rad}$ (i.e. 4-19 pixels across) from Mars at its farthest and closest points from Earth respectively, the center of the Earth image can be well resolved at the sub-arcsecond level. In planetary orbit or during cruise phase, the transponder CCD sees a rather bright sunlit Earth against a dark background except when $\alpha_{\mathrm{A}} \sim 0^{\circ}$ or $180^{\circ}$. However, even when the Earth's "nightside" is largely directed toward the transponder, there can be sufficient forward scattering of solar light by the Earth's atmospheric rim for detection (Mallama, 1998).

Since acquisition of planetary and lunar images against a dark sky is relatively easy, we only need to concern ourselves with daylight acquisition. Since the Earth and Moon have the same solar aspect angle when viewed from Mars, we can use the same expression to compute the lunar contrast (excluding rare eclipse events). The worst case noise scenario is for a transponder on the planet's surface operating in daylight in the presence of a scattering atmosphere. Let us assume that both terminals contain a square CCD array containing $N_{\text {pix }}$ pixels and that the Earth image does not move substantially during the CCD integration time. The number of pixels illuminated by the partially sunlit Earth image (Planet A) is approximately

$$
N_{\mathrm{A}} \cong \pi\left(\frac{r_{\mathrm{A}}}{R}\right)^{2} \frac{N_{\mathrm{pix}}}{\Omega_{\mathrm{ccd}}^{\mathrm{B}}} f_{\mathrm{A}},
$$

where $\Omega_{\text {ccd }}^{\mathrm{B}}$ is the solid angle viewed by the Terminal B CCD array, $f_{\mathrm{A}}$ represents the fraction of the Earth disk area illuminated by the Sun where 
MIGROLASER ALTIMETER TRANSPONDER (MAT) BLOCK DIAGRAM

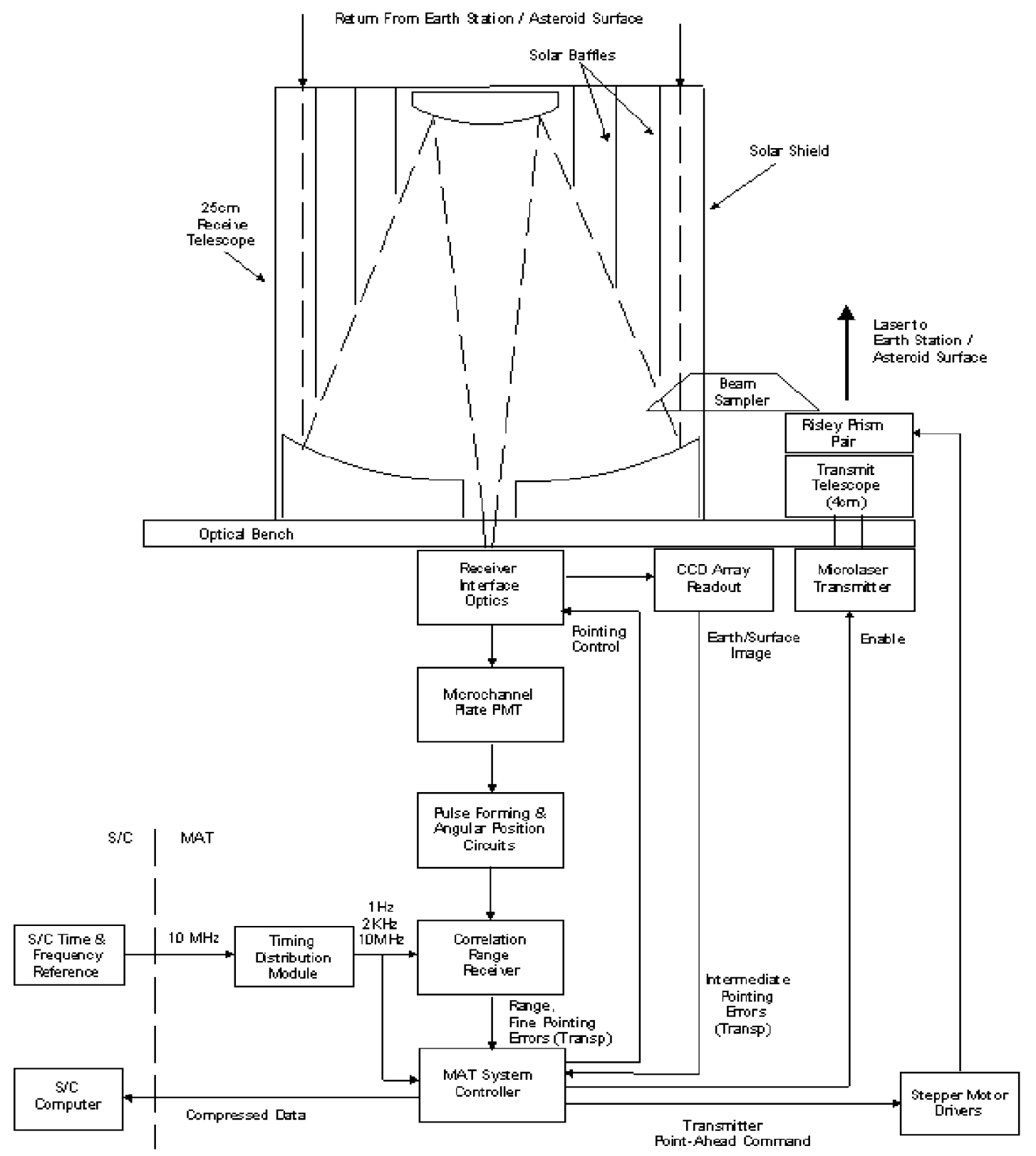

Fig. 7. Block diagram of a dual-mode Microlaser Altimeter Transponder (MAT) instrument. The CCD array is used to crudely center the planetary image within the quadrant photomultiplier FOV so that the latter can provide subarcsecond pointing corrections to maximize the link. A Risley prism pair is used to offset the transmitter line of sight for "pointahead". The accuracy of the point-ahead can be monitored by leaking a small portion of the outgoing pulse into the receiver and onto the CCD. The transponder can be used at any point during the mission, i.e. interplanetary cruise, in orbit, or the surface of another planet. While still in orbit, the same system can produce accurate 3-D topographic maps of the surface prior to landing and record coaligned 2-D visual images using the high resolution CCD camera. 


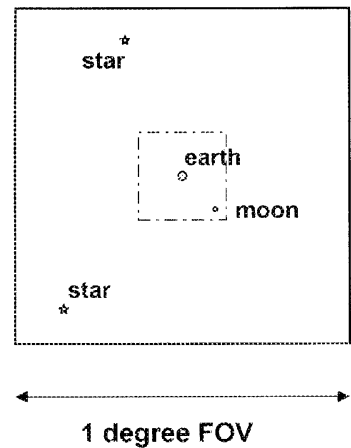

(a)

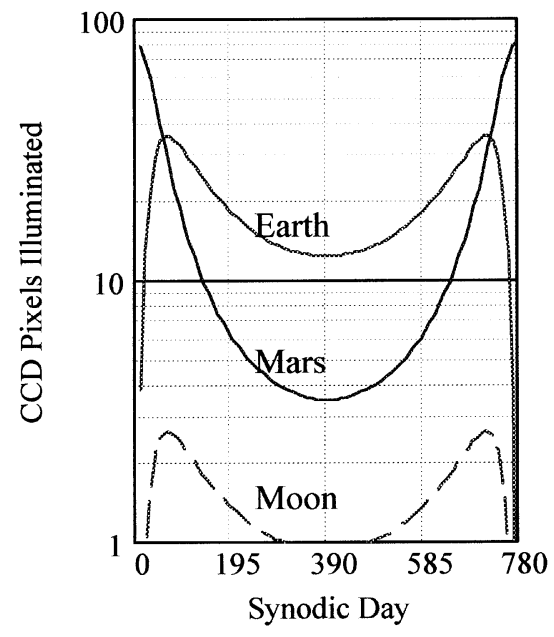

(c)

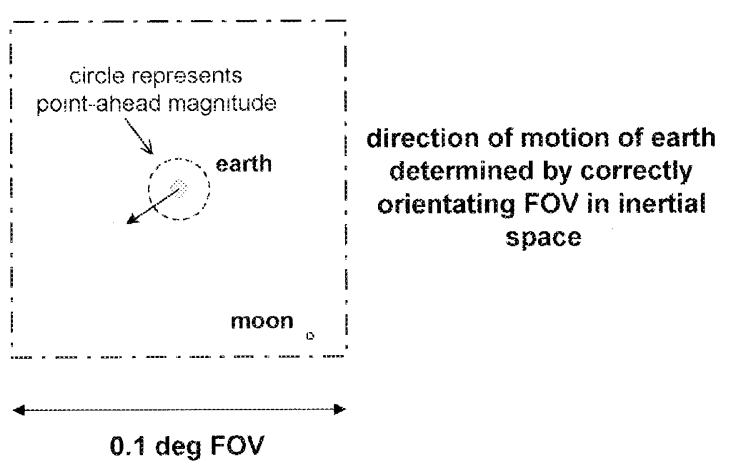

(b)

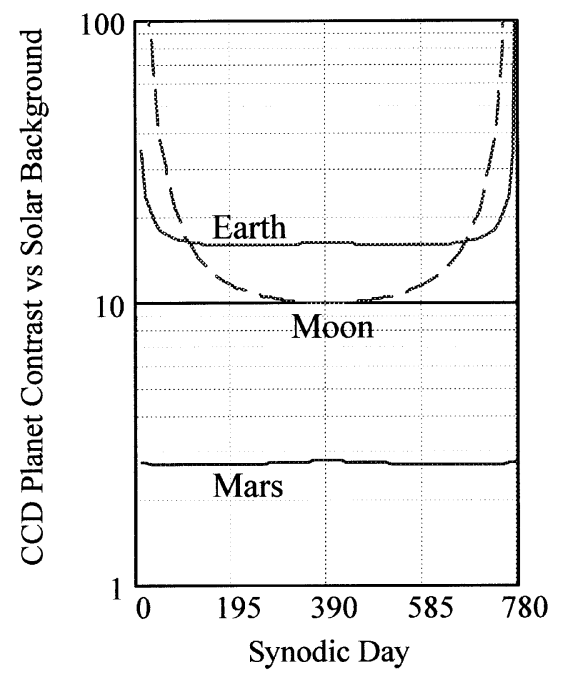

(d)

Fig. 8. (a) Simulated CCD camera display of a $1^{\circ}$ by $1^{\circ}$ FOV showing the centered Earth, Moon, and background stars used in the computation of the transmitter point ahead angle and direction. (b) The circle around the Earth represents the magnitude of the angular offset as computed from the planetary ephemerides, and the arrow represents the Earth's forward motion as determined by other celestial objects (Moon, stars) in the camera FOV. The intersection of these two curves indicates the desired laser beam pointing. Sampling of the outgoing laser beam by the prism assembly can provide pointing verification. (c) Number of CCD pixels illuminated at the viewing terminal by the Earth, Mars, and Moon as a function of synodic day; (d) Worst-case contrast of planetary image relative to background counts caused by solar scattering from the local atmosphere during daylight operations.

$$
\begin{aligned}
& f_{\mathrm{A}}\left(0 \leqslant \alpha_{\mathrm{A}} \leqslant \frac{\pi}{2}\right)=\left|\cos \alpha_{\mathrm{A}}\right|^{3} \\
& +\frac{2}{\pi}\left\{\int_{0}^{\sin \alpha_{\mathrm{A}}} \mathrm{d} x \sqrt{\sin ^{2} \alpha_{\mathrm{A}}-x^{2}}+\left|\cos \alpha_{\mathrm{A}}\right| \int_{\left|\cos \alpha_{\mathrm{A}}\right|}^{1} \mathrm{~d} x x \cos ^{-1}\left[-\cot \alpha_{\mathrm{A}} \frac{\sqrt{1-x^{2}}}{x}\right]\right\}
\end{aligned}
$$




$$
\begin{aligned}
& f_{\mathrm{A}}\left(\frac{\pi}{2} \leqslant \alpha_{\mathrm{A}} \leqslant \pi\right) \\
& =\frac{2}{\pi}\left\{\int_{0}^{\sin \alpha_{\mathrm{A}}} \mathrm{d} x \sqrt{\sin ^{2} \alpha_{\mathrm{A}}-x^{2}}+\left|\cos \alpha_{\mathrm{A}}\right| \int_{\left|\cos \alpha_{\mathrm{A}}\right|}^{1} \mathrm{~d} x x \cos ^{-1}\left[-\cot \alpha_{\mathrm{A}} \frac{\sqrt{1-x^{2}}}{x}\right]\right\}
\end{aligned}
$$

and $\alpha_{\mathrm{A}}$ is once again the aspect angle in Fig. 3. The number of illuminated pixels as a function of the synodic phase is illustrated in Fig. 8c for terminals operating at Earth and Mars. The Moon has the same aspect angle as Earth when viewed from Mars, but the image occupies fewer pixels due to its smaller size.

The count rate due to solar scatter from the local atmosphere is distributed over all of the pixels whereas the solar radiation scattered from the opposite planet is distributed only over $N_{\mathrm{A}}$ pixels. We can now use (13) and (14) to obtain an expression for the CCD contrast of the planetary image at terminal $\mathrm{B}$

$$
\begin{aligned}
C_{\mathrm{CCD}}^{\mathrm{B}} & =1+\frac{N_{\mathrm{pix}}}{N_{\mathrm{A}}} \underset{\frac{n_{\mathrm{sa}}^{\mathrm{B}}}{n_{\mathrm{ls}}^{\mathrm{B}}}}{=}=1+\frac{4}{f_{\mathrm{A}}}\left(\frac{R_{\mathrm{B}}}{R_{\mathrm{A}}}\right)^{2} \frac{\left[\rho_{\mathrm{A}} g_{\mathrm{ps}}^{\mathrm{B}}\left(\alpha_{\mathrm{A}}\right)+\frac{1}{2} \ln \left(\frac{1}{T_{\mathrm{A}}}\right) g_{\mathrm{as}}^{\mathrm{B}}\left(\alpha_{\mathrm{A}}\right)\right]}{\sec \theta_{B}\left(\frac{1-T_{\mathrm{B}}^{\sec \theta_{s}-\sec \theta_{\mathrm{B}}}}{\sec \theta_{s}-\sec \theta_{\mathrm{B}}}\right)} \\
& \simeq 1+\frac{4}{f_{\mathrm{A}}}\left(\frac{R_{\mathrm{B}}}{R_{\mathrm{A}}}\right)^{2} \frac{\left[\rho_{\mathrm{A}} g_{\mathrm{ps}}^{\mathrm{B}}\left(\alpha_{\mathrm{A}}\right)+\frac{1}{2} \ln \left(\frac{1}{T_{\mathrm{A}}}\right) g_{\mathrm{as}}^{\mathrm{B}}\left(\alpha_{\mathrm{A}}\right)\right]}{\ln \left(\frac{1}{T_{\mathrm{B}}^{\sec \theta_{\mathrm{B}}}}\right)}
\end{aligned}
$$

The contrast of the planetary images against the solar-illuminated atmosphere during daylight operations on Planet B's surface is given by (29); the corresponding equation for the opposite terminal given by an interchange of $\mathrm{A}$ and $\mathrm{B}$ in that expression. Fig. 8d plots the contrast of the Mars, Earth, and Moon images against the scattering of solar radiation by the local atmosphere during daylight operations as a function of the synodic phase.

We now want to integrate the CCD scene long enough so that we have a high probability of correctly identifying the planetary image. We can use a variation of the Differential Cell Count scheme described in Section 3.3 to derive an optimum pixel threshold given by

$$
K_{\mathrm{CCD}}^{\mathrm{B}}=\frac{\left(C_{\mathrm{CCD}}^{\mathrm{B}}-1\right) N_{\mathrm{ls}}^{\mathrm{B}}+\ln \left(\frac{N_{\text {pix }}}{N_{\mathrm{A}}}-1\right)}{\ln \left(C_{\mathrm{CCD}}^{\mathrm{B}}\right)}
$$

where $N_{\text {pix }}$ is the total number of pixels in the CCD array, $N_{\mathrm{A}}$ is the number of pixels illuminated by the planetary image as given by (27), and 


$$
\begin{aligned}
N_{\mathrm{ls}}^{\mathrm{B}} & =\frac{\dot{n}_{\mathrm{l}}^{\mathrm{B}} \tau_{I}}{N_{\mathrm{pix}}}=\frac{\eta_{\mathrm{ccd}}^{\mathrm{B}} \eta_{r^{\prime}}^{\mathrm{B}}}{h v} \frac{N_{\lambda}^{\mathrm{B}}\left(\Delta \lambda_{\mathrm{ccd}}^{\mathrm{B}}\right) A_{\mathrm{B}} \Omega_{\mathrm{ccd}}^{\mathrm{B}} \tau_{I}}{4 \pi N_{p i x}}\left\{\sec \theta_{\mathrm{B}} T_{\mathrm{B}}^{\mathrm{sec} \theta_{\mathrm{B}}}\left[\frac{1-T_{\mathrm{B}}^{\sec \theta_{s}-\sec \theta_{\mathrm{B}}}}{\sec \theta_{S}-\sec \theta_{\mathrm{B}}}\right]\right\} \\
& \simeq \frac{\eta_{\mathrm{ccd}}^{\mathrm{B}} \eta_{r^{\prime}}^{\mathrm{B}}}{h v} \frac{N_{\lambda}^{\mathrm{B}}\left(\Delta \lambda_{\mathrm{ccd}}^{\mathrm{B}}\right) A_{\mathrm{B}} \Omega_{\mathrm{ccd}}^{\mathrm{B}} \tau_{I}^{\mathrm{B}}}{4 \pi N_{p i x}}\left[T_{\mathrm{B}}^{\sec \theta_{\mathrm{B}}} \ln \left(T_{\mathrm{B}}^{\mathrm{sec} \theta_{\mathrm{B}}}\right)\right]
\end{aligned}
$$

is the mean noise count per pixel due to solar scattering off the atmosphere collected during an integration time, $\tau_{I}$. In deriving (31), we have used (19) and allowed the CCD quantum efficiency $\eta_{c c d}^{B}$, the optical throughput $\eta_{r}^{\mathrm{B}}$, field of view $\Omega_{\mathrm{ccd}}^{\mathrm{B}}$, and spectral bandpass $\Delta \lambda_{\text {ccd }}^{\mathrm{B}}$ of the CCD channel to be different from the range receiver channel. Since the CCD spectral bandpass filter acts equally on the planetary albedo "signal" and the solar scattering "noise", the contrast of the planetary image (unlike the ranging signal) is unaffected by our choice of spectral bandwidth. Furthermore, the effective differential pixel count will asymptotically approach unity as the mean noise cell count $N_{\mathrm{ls}}^{\mathrm{B}}$ increases, and we should therefore choose a large spectral bandwidth filter and the longest CCD integration time consistent with the loop bandwidth required to keep the planetary image centered within the CCD array.

Once the Earth is centered in the receiver FOV, receipt of laser pulses from Earth is ensured provided: (1) the Earth ground station pointing error is less than the ground laser beam divergence (nominally about $50 \mu \mathrm{rad}$ ); (2) the range receiver FOV is larger than the Earth disk and is adequately boresighted with the CCD array; and (3) there is sufficient signal to surpass the detection threshold of the receiver. Angular errors due to uncertainties in planetary and most other ephemerides to important bodies in the inner Solar System are typically far smaller $(<0.01$ $\mu \mathrm{rad})$ than the nominal laser beam divergence assumed here $(50 \mu \mathrm{rad})$ as are the pointing control errors in a star-calibrated, meter-class telescope/tracking system $(<15 \mu \mathrm{rad})$. Furthermore, arriving laser photons can be detected by a quadrant ranging detector, which is co-aligned with the center of the CCD array. As in NASA's developmental SLR2000 satellite laser ranging station, the quadrant detector permits fine pointing corrections of the transponder receiver at the subarcsecond level (Degnan and McGarry, 1997).

\subsection{Transmitter point-ahead}

In a general transponder experiment, the CCD Earth image tells us where the Earth was situated one transit time earlier whereas the narrow beam transmitter must be pointed to where the Earth will be one transit time later. Thus, one transit time later and in the image plane of our receiver CCD, the Earth will lie somewhere on the perimeter of a circle centered on our Earth image and having an angular radius equal to the differential pointing angle. A method of computing the non-relativistic transmitter point-ahead is provided in Appendix B. The projection of the Earth's forward velocity vector in the receiver plane is then needed to determine the precise point on the circle to which the laser beam should be directed. The forward velocity vector can be inferred from simultaneous CCD observations of the Moon and/or stars within the camera FOV. The Moon is expected to be visible about $80 \%$ of the time (Mallama, 1998) and, when it is not, background stars within the nominal $1^{\circ} \times 1^{\circ} \mathrm{CCD}$ FOV can be substituted as available.

Differential pointing of the transmitter can be most easily accomplished in a bistatic optical system with a pair of azimuthally driven Risley prisms in the transmit path as in Fig. 7 . The 
magnitude of the angular offset is determined by the relative orientations of the prisms (which remains fairly constant over long periods of time - see Fig. 9e), and the direction is changed by driving them in unison to locate the precise point on the circle. As in Fig. 7, transmitter feedback to the receiver can be provided by a prism or two mirror reflector which samples the beam as it exits the transmit telescope and reflects it into the receiver for time logging and/or verification of
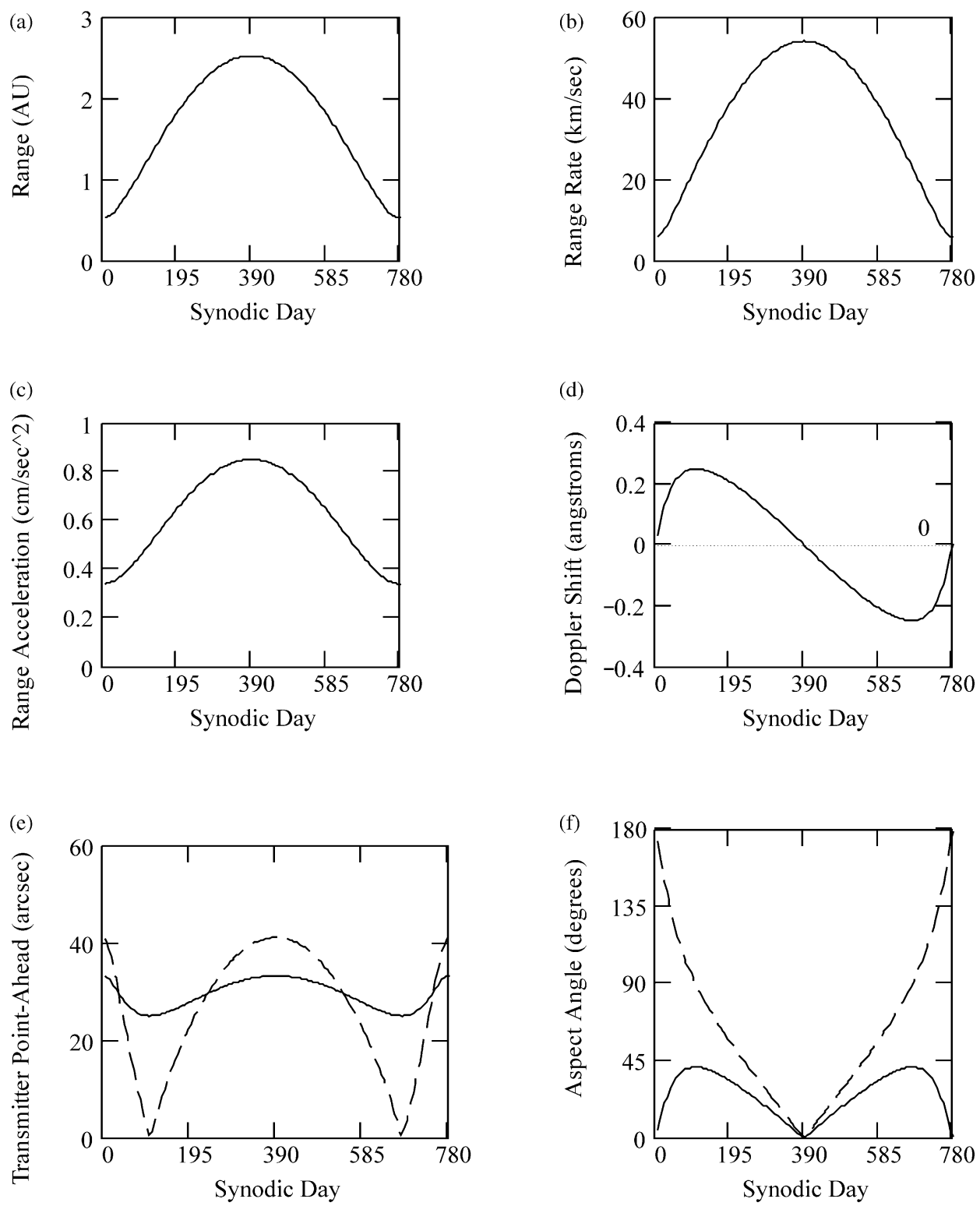

Fig. 9. Various plots relevant to the Earth-Mars link as a function of synodic phase/day: (a) range in AU; (b) rangerate in $\mathrm{km} / \mathrm{s}$; (c) range acceleration in $\mathrm{cm} / \mathrm{s}^{2}$; (d) Doppler shift in Angstroms; (e) transmitter point ahead angle in arcseconds; and (f) aspect angles. In (e) and (f), the Earth terminal is represented by a solid line and the Mars terminal by a dashed line. 
the transmit angle. As mentioned previously, the bistatic optical design also helps to eliminate laser backscatter from the local atmosphere as an important noise source.

\subsection{Acquisition and tracking of the opposite terminal in range space}

For the moment, we will assume that both terminals have approximately the same laser fire interval, $\tau_{\mathrm{qs}}$. During each interval or cycle, each terminal records the time of departure of the outgoing pulse and the time of arrival of any incoming pulses relative to a fixed sub-clock, which is counting at the nominal laser fire rate and is derived from a highly accurate frequency standard such as a rubidium or cesium clock. In the case of a Mars lander, an onboard knowledge of the Earth and Mars ephemerides and planetary spin axis orientation and rotation rates permits a fair a priori estimate of the interplanetary distance (typically within a few tens of kilometers), the differential pointing angle (within an arcsecond) and the forward direction of the Earth's motion. If the distance between terminals can be known a priori to well within the distance light travels in one laser fire interval, the incoming pulse can be easily matched up with the proper cycle at the opposite terminal where it originated. Because light transit times within the inner Solar System can span several tens of minutes, there may be thousands to millions of cycles between the matching cycles for each terminal, depending on the laser fire rate and the distance between terminals. During initial acquisition, the uncertainty in the laser time of fire at the opposite terminal can be as large as the laser fire interval itself if the clocks at Terminals A and B have not been compared in some time and are totally uncorrelated. Once the signal from the opposite terminal is acquired, however, this uncertainty shrinks rapidly, allowing the receivers to independently narrow their range gates for improved noise rejection.

In a satellite laser ranging (SLR) system, the range between ground station and satellite changes rapidly and the position of the range gate is varied in real time, based on an a priori range estimate, in an attempt to keep the satellite return centered in the gate. The measured pulse times of flight, displayed on the ordinate axis relative to the center of the range gate versus elapsed time on the abscissa, is referred to as an Observed Minus Calculated (O-C) plot (Degnan, 1985). If our a priori range and range rate corrections and satellite force models were perfect and additionally there were no range or time biases in the computed orbit, the corresponding "Observed Minus Calculated" $(\mathrm{O}-\mathrm{C})$ curve would place all of the observed signal photons within a single range bin centered in the range gate. In this highly idealized example, the temporal width of the signal data distribution would then be determined by the timing precision of the range receiver and small atmosphere-induced fluctuations which, in modern SLR systems, is characterized by a one sigma RMS single-shot range scatter of one cm (67 ps) or less (Degnan, 1993). Background photons and detector dark counts, on the other hand, are randomly distributed throughout the entire range gate. Thus, when histogrammed according to the individual range bins, the signal counts stand out from the background due to their high "temporal" correlation in the range axis (Degnan, 2001). In actual SLR experiments, range biases displace the signal from the center of the range window, and orbital time biases introduce a slope in the signal data as viewed in an $\mathrm{O}-\mathrm{C}$ plot. Once a slope is observed in the data, however, a time bias correction can be iteratively applied in the range model as necessary to reduce the slope and ultimately the size of the range bin and/or gate for better signal contrast and noise rejection.

A similar slope will occur in an asynchronous transponder link when an interplanetary range-rate, estimated from planetary ephemerides and applied to the photon arrival times, has a residual 
error. As previously mentioned in Section 2.1, the error in range rate computed from contemporary ephemerides for Earth and Mars is estimated to be less than $1 \mathrm{~cm} / \mathrm{s}$ (Lemoine, 2000), corresponding to a very shallow slope of less than $33 \mathrm{ps} / \mathrm{s}$ in the $\mathrm{O}-\mathrm{C}$ curve. An additional component of range rate results from the rotation of the planets about their respective axes. For Planet A, it is a sinusoidal function with peak amplitude

$$
\left|r_{\mathrm{A}}\right|_{\max }=\varpi_{\mathrm{A}} r_{\mathrm{A}} \cos \lambda_{\mathrm{A}} \cos i_{\mathrm{A}}
$$

where $\varpi_{\mathrm{A}}$ is the angular rotation rate about the axis, $r_{\mathrm{A}}$ is the volumetric mean radius of Planet $\mathrm{A}, \lambda_{\mathrm{A}}$ is the latitude of the station, and $i_{\mathrm{A}}$ is the inclination of the spin axis to the orbital plane. An identical expression applies to the station on Planet B. Using the constants in Table 1, the maximum range rate introduced by the rotation of Earth and Mars for equatorial stations is 67.6 and $34.6 \mathrm{~m} / \mathrm{s}$ respectively and decreases with latitude. Thus, Eq. (32) results in $\mathrm{O}-\mathrm{C}$ slopes between 0 and $230 \mathrm{~ns} / \mathrm{s}$ due to Earth rotation and between 0 and $120 \mathrm{~ns} / \mathrm{s}$ due to Mars rotation. These are large compared to the residual slope after correction for interplanetary motion and suggest that an a priori correction for planetary rotation be applied to the raw data prior to producing the $\mathrm{O}-\mathrm{C}$ histogram plot.

Double-ended transponder systems use two clocks and two lasers whereas a single-ended SLR system uses only one laser and one clock in recording the pulse time of flight, and this difference leads to some apparent range-rate errors as recorded in a transponder $\mathrm{O}-\mathrm{C}$ plot. For example, if the frequency standards (clocks) at the two interacting transponder terminals have a frequency offset, there will be an additional contribution to the observed slope in $\mathrm{O}-\mathrm{C}$ space given by the familiar Doppler equation, i.e.

$$
\sigma_{c}=-c \frac{\Delta f}{f_{c}}
$$

where $f_{c}$ is the nominal clock frequency at both terminals and $\Delta f$ is the offset frequency between the clocks as discussed in Section 2.3. In addition to normal clock drift, there are additional shifts due to the unequal relativistic effects of velocity and gravity at the two terminals, but these are generally quite small and calculable. A fractional clock offset of $1 \times 10^{-11}$ corresponds to a range rate error in $\mathrm{O}-\mathrm{C}$ space of less than $3 \mathrm{~mm} / \mathrm{s}$, or about $20 \mathrm{ps} / \mathrm{s}$, and is comparable in magnitude to the residual range-rate error from the ephemerides. This level of offset or smaller is easily achieved by a rubidium or cesium standard, but, for an undisciplined quartz crystal oscillator, the slope could be up to two orders of magnitude higher. We can now see that our original assumption of approximately equal fire rates is not a requirement for signal recovery; it is only necessary to know the fire rate of the opposite terminal and that it is driven by a stable frequency standard.

A further difference with SLR arises from the use of two lasers. In a single-ended SLR system, the RMS scatter of the data depends on the jitter in the detector response, the resolution of the timer, and the impulse response of the satellite. The jitter in the laser fire time, however, cancels out of the range measurements since the start and stop pulses input to the interval timer are affected equally. In a two-way asynchronous transponder, on the other hand, the unequal jitter in the fire times of the two lasers leads to an additional random range error, which will significantly broaden the signal data in the $\mathrm{O}-\mathrm{C}$ plot relative to the single laser case. 
Any uncorrected slope or breadth to the signal data must be accommodated by a larger range bin, $\tau_{b}$, which in turn increases the scale energy via (22), (24), and (25). The breadth or precision of the signal data sets a lower limit on the range bin size, even for automated high SNR single pulse detection. With a residual data slope, any increase in the receiver integration (frame) time in a low SNR photon-counting system must be accompanied by a proportional increase in the range bin width to ensure that we capture all or most of the signal photons from multiple laser fires in a single cell. One rule of thumb that appears to work well in simulations is to choose a bin size at least twice as large as the expected overall RMS variation in the $\mathrm{O}-\mathrm{C}$ range data over a frame, i.e.

$$
\tau_{b} \geqslant 2 \sqrt{\left(\left\langle\sigma_{e}^{2}\right\rangle+\left\langle\sigma_{r}^{2}\right\rangle+\left\langle\sigma_{c}^{2}\right\rangle\right) \tau_{\mathrm{F}}^{2}+\left\langle\tau_{\mathrm{A}}^{2}\right\rangle+\left\langle\tau_{\mathrm{B}}^{2}\right\rangle}
$$

where $\left\langle\sigma_{e}^{2}\right\rangle,\left\langle\sigma_{r}^{2}\right\rangle$, and $\left\langle\sigma_{c}^{2}\right\rangle$ are the expected variances in the residual slopes in $\mathrm{O}-\mathrm{C}$ space due to errors in ephemerides, planetary rotation rates, and clock offsets respectively, $\tau_{\mathrm{F}}$ is the frame time, and $\left\langle\tau_{\mathrm{A}}^{2}\right\rangle$ and $\left\langle\tau_{\mathrm{B}}^{2}\right\rangle$ are the variances in the recorded fire times (including laser, detector and timer jitter) at Terminals A and B respectively. Variances in the relativistic corrections can be included in (34) as well.

\subsubsection{Summary of range acquisition procedure}

To summarize, the search in the third dimension, range, is carried out independently by each terminal by: (1) breaking up the laser fire interval (or some smaller range window depending on the level of our a priori knowledge) into appropriately sized range bins; (2) applying corrections to the raw photon arrival times using a priori information (primarily range rate) to form an $\mathrm{O}-\mathrm{C}$ plot; (3) binning and histogramming these corrected ranges; (4) applying a threshold test to each bin/cell to determine candidate signal cells; and (5) ultimately applying a software filter to make a final selection of signal cells, reject the noise cells, calculate and correct for any residual range-rate error to further improve the signal contrast, and narrow the range bin/window for better noise rejection. In prior publications (Degnan and McGarry, 1997; Degnan, 2002), we have referred to the combination of hardware and software that performs these functions as a Correlation Range Receiver $(C R R)$. Post-Detection Poisson Filters and Correlation Range Receivers, as they apply to laser altimeters, are discussed in depth in a companion article in this journal (Degnan, 2002). Such filters tend to be simpler and even more effective when used in single-ended ranging or double-ended transponder systems because, as mentioned previously, the corrected signal data falls along a straight line in $\mathrm{O}-\mathrm{C}$ space whereas surface topography results in a confined but random path over short time intervals.

\section{Earth-Mars transponder link analysis}

Table 1 summarizes some planetary characteristics relevant to the link analysis. As mentioned in the introduction, we are interested in order of magnitude results, and we will therefore ignore the small orbital eccentricities (0.0935 for Mars and 0.0167 for Earth) and small relative tilt $\left(\sim 1.85^{\circ}\right)$ in the planetary orbital planes and assume coplanar (xy plane), circular, and coaxial 
orbits of Planets $\mathrm{A}$ and $\mathrm{B}$ about the Sun having radii $R_{\mathrm{A}}$ and $R_{\mathrm{B}}$ respectively. We will also ignore any relativistic corrections. In this approximation, the vector positions of Planet A and B at any given time are given by the simple formulae

$$
\left|\begin{array}{l}
x_{\mathrm{A}, \mathrm{B}} \\
y_{\mathrm{A}, \mathrm{B}}
\end{array}\right|=R_{\mathrm{A}, \mathrm{B}}\left|\begin{array}{c}
\cos \omega_{\mathrm{A}, \mathrm{B}} t \\
\sin \omega_{\mathrm{A}, \mathrm{B}} t
\end{array}\right|
$$

where $\omega_{\mathrm{A}}$ and $\omega_{\mathrm{B}}$ days are the orbital angular frequencies for the Earth and Mars respectively, and the time origin is arbitrarily chosen when Planets A and B are at the Point of Closest Approach (PCA) on the same side of the Sun. The planetary positions repeat themselves with an angular synodic frequency given by

$$
\omega_{\mathrm{S}}=\omega_{\mathrm{A}}-\omega_{\mathrm{B}}=2 \pi\left(\frac{1}{\tau_{\mathrm{A}}}-\frac{1}{\tau_{\mathrm{B}}}\right)=\frac{2 \pi}{\tau_{\mathrm{S}}}
$$

where $\tau_{\mathrm{A}}$ and $\tau_{\mathrm{B}}$ are the orbital periods of Planets $\mathrm{A}$ and $\mathrm{B}$ about the Sun and $\tau_{\mathrm{S}}$ is the synodic period which, for Earth and Mars, corresponds to approximately 780 days.

It is worth noting that the interplanetary range rate introduces a variable Doppler shift in the laser wavelength as viewed by the opposite terminal, given by

$$
\Delta \lambda(t)=\frac{\lambda \stackrel{\bullet}{c}}{c}(t) \bullet \frac{\vec{R}(t)}{R(t)}
$$

where

$$
\vec{R}(t)=\vec{R}_{\mathrm{B}}(t)-\vec{R}_{\mathrm{A}}(t)
$$

is the instantaneous interplanetary vector. The Doppler shift sets a lower limit on the bandwidth of a fixed spectral filter used in the transponder receivers although tunable tracking filters are possible.

In Fig. 9, we plot: (a) the Earth-Mars range, (b) range-rate, (c) range acceleration, (d) Doppler shift, (e) point-ahead angles (see Appendix B), and (f) the aspect angles as a function of the synodic phase (day) for the Earth-Mars transponder link. Table 1 summarizes the key planetary parameters. The maximum range-rate of approximately $55 \mathrm{~km} / \mathrm{s}$ and range acceleration of 8.2 $\mathrm{mm} / \mathrm{s}^{2}$ both occur midway in the synodic period at the point of maximum range (2.52 AU). The Doppler-induced wavelength shift is confined to a range of about $\pm 0.25 \AA$. From Fig. 9e, the transmitter point-ahead for the Earth station varies between about 25 and 33 arcseconds and is a maximum at the points of minimum and maximum range. For the Mars station, the curve has the same peaks and valleys, but the point-ahead angle varies over a much wider range, between 0 and 41 arcseconds. From Fig. 9f, we see that Mars is always at least partially illuminated when viewed from Earth while Earth, as viewed from Mars, runs the entire gamut from being backlit at the point of minimum interplanetary range to fully illuminated at the point of maximum range. 


\subsection{High SNR example: NASA MOBLAS station ranging to a "Green MOLA"}

Our first link example will be a conventional high Signal-to-Noise Ratio (SNR) link between a NASA Mobile Laser (MOBLAS) station (Degnan, 1985) and a $532 \mathrm{~nm}$ (green), subnanosecond version of the Mars Orbiter Laser Altimeter (MOLA) instrument (Smith et al., 1999), both of which are operating at a zenith angle of $30^{\circ}$. The laser energy of the "green MOLA" was chosen to provide a balanced transponder link with MOBLAS assuming the $50 \mathrm{~cm}$ MOLA receive aperture. Coincidentally, the green pulse energy of $43 \mathrm{~mJ}$, multiplied by the MOBLAS repetition rate of $5 \mathrm{~Hz}$ yields an average power of $215 \mathrm{~mW}$ which is roughly half the $1064 \mathrm{~nm}$ power produced by the MOLA instrument at $10 \mathrm{~Hz}$. Thus, the reduction in repetition rate compensates for the roughly $50 \%$ loss in laser power due to conversion of the fundamental Nd:YAG wavelength from 1064 to $532 \mathrm{~nm}$ so that the "green MOLA" should consume roughly the same prime power as the original MOLA. In both systems, we assume an improved transmitter beam divergence of $50 \mu \mathrm{rad}$ with a somewhat wider receiver FOV of $100 \mu \mathrm{rad}$ in order to accommodate residual boresight errors/drifts between the transmitter and receiver. Table 2 summarizes the assumed instrumental parameters for the high SNR link.

In this high SNR example, we assume that each terminal detects single subnanosecond pulses, i.e. one laser fire per cell. We therefore select a range bin size, $\tau_{\mathrm{b}}=1 \mathrm{~ns}$, which comfortably accomodates all of the signal photons in a single pulse. We further assume the worst case scenario of totally unsynchronized clocks at Terminals A and B so that the receiver is initially ungated for the entire interpulse period of $200 \mathrm{~ms}$. This results in a very large number of range bins, i.e. $N_{\text {bin }}=2 \times 10^{8}$. Using (18) through (21), we plot in Fig. 10: (a) the optimum threshold; (b) the differential cell count; (c) the probability of correctly detecting the signal cell; and (d) the mean false alarms per laser fire for the Earth and Mars terminals as a function of the synodic phase for both night and day operations.

During the first quarter of the synodic cycle, the optimum threshold drops from roughly 15-18 pe to 3-4 pe then levels off for two quarter cycles before climbing again to the 15-18 pe level during the last quarter. Near minimum interplanetary range, the signal is higher and the optimum threshold tends to higher values so that the differential cell count algorithm can suppress the number of false alarms to a negligible number without significantly affecting the signal detection probability. Near maximum range, the algorithm lowers the threshold to allow a higher probability of detection for the weaker signal but in the process makes false alarms more likely. The probability of successfully detecting the signal is essentially unity for the first and last quarter cycle for both terminals under both day and night conditions. A negligible number of false alarms occur $(<1$ per fire) under both day and night scenarios during the first and last quarters. Even near the point of maximum interplanetary range, the detection probability for daylight operations drops to about $86 \%$ for the Earth terminal and $90 \%$ for the Mars terminal. Furthermore, because of the lowered threshold near the point of maximum range and the huge number of $1 \mathrm{~ns}$ range bins within the ungated laser fire period of $200 \mathrm{~ms}$, the mean false alarms can jump to about 1 in 1000 frames at Earth and to about 1 in 15 frames at Mars in spite of an extremely low false alarm probability per bin which is on the order of $5 \times 10^{-8}$ for the Earth terminal and $5 \times 10^{-7}$ for the Mars terminal. Nevertheless, the number of false alarms is still small compared to the number of signal returns. Furthermore, the false alarms are widely scattered throughout the laser fire interval whereas the $5 \mathrm{~Hz}$ signal counts will stand out prominently against the false 


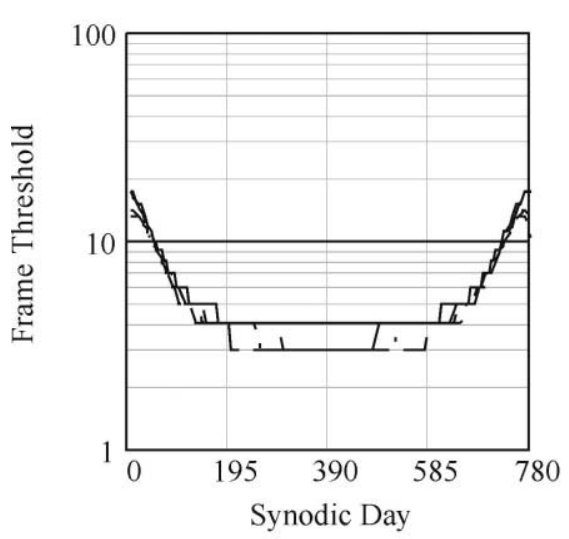

(a)

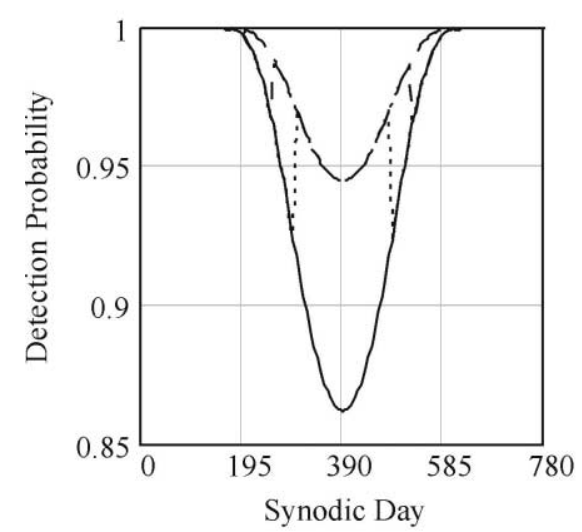

(c)

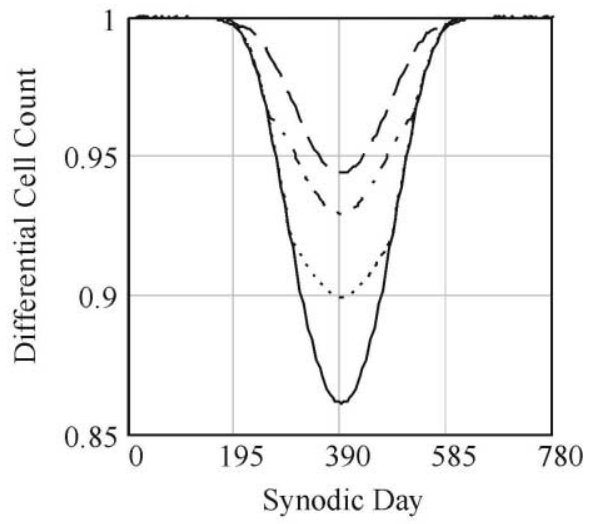

(b)

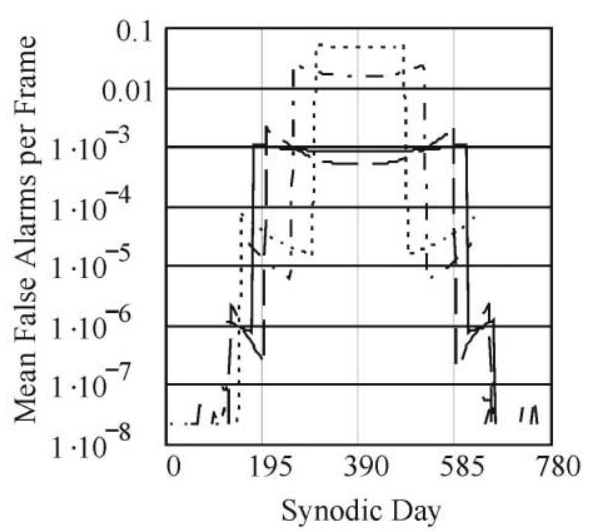

(d)

Fig. 10. Operating parameters as a function of synodic phase/day for the balanced, high SNR Earth-Mars transponder link operating at $5 \mathrm{~Hz}$ during initial acquisition under worst case conditions of unsynchronized clocks: (a) optimum detection threshold; (b) optimized differential cell count; (c) optimized signal detection probability; and (d) mean false alarms per laser fire. Earth daylight and night operations are indicated by the solid and dashed lines respectively; Mars daylight and night operations are represented by dotted and dash-dotted lines respectively.

counts when displayed in a histogrammed $\mathrm{O}-\mathrm{C}$ plot because of their temporal coherence. Furthermore, once the signal is acquired, the range gate can be narrowed sufficiently to suppress virtually all subsequent false alarms.

\subsection{Low SNR example: SLR2000 ranging to a "Green MLA"}

We now provide an example of a low SNR link where multiple single photon returns in a high repetition rate link are accumulated in the cells of a correlation range receiver. The potential advantages of such a system have been described previously (Degnan et al., 1998; Degnan, 2001). The Earth ground station is assumed to be NASA's developmental SLR2000 station (Degnan and McGarry, 1997) and the Mars terminal is assumed to be similar in size to the Messenger 


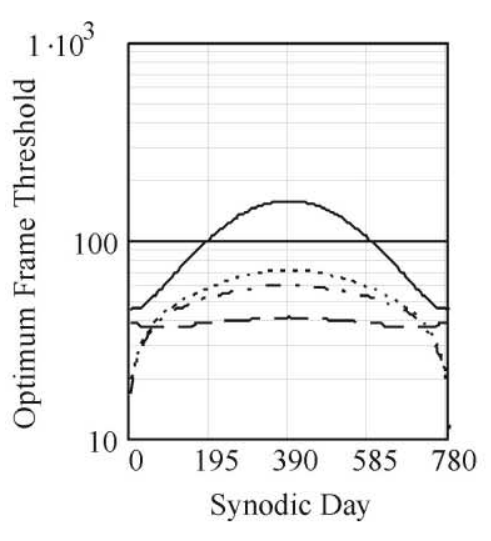

(a)

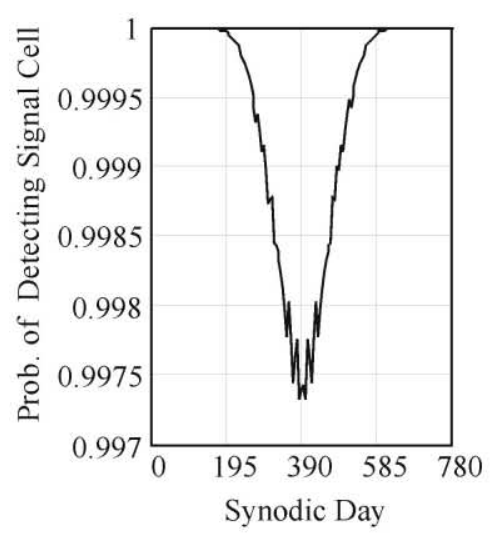

(c)

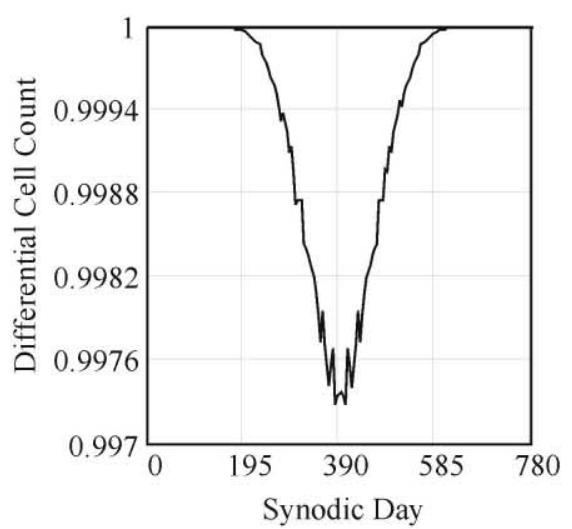

(b)

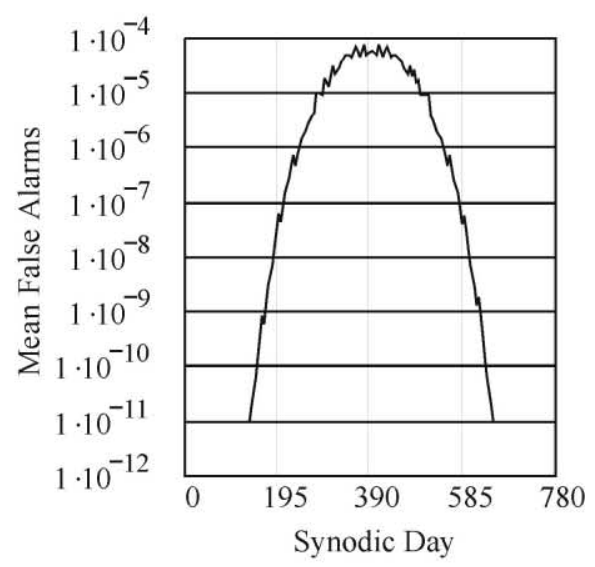

(d)

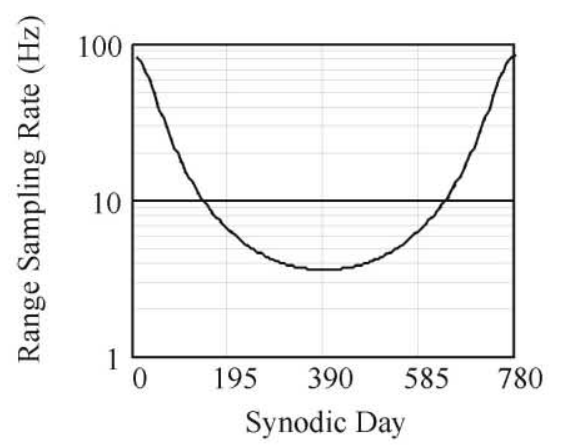

(e)

Fig. 11. Operating parameters as a function of synodic phase/day for the balanced, low SNR, photon-counting EarthMars transponder link operating at $2 \mathrm{kHz}$ during initial acquisition under worst case conditions of unsynchronized clocks: (a) optimum detection threshold; (b) optimized differential cell count; (c) optimized signal detection probability; (d) mean false alarms per laser fire; and (e) range sampling rate. In (a), Earth daylight and night operations are indicated by the solid and dashed lines respectively whereas Mars daylight and night operations are represented by dotted and dash-dotted lines respectively. Because the link is "balanced", the four curves in (b) through (d) overlap. 
Mercury Laser Altimeter (MLA), which is smaller and more lightweight than the Mars MOLA instrument. Both instruments are assumed to use compact, low energy, microlaser transmitters which operate at a much higher $2 \mathrm{kHz}$ rate (Degnan and Zayhowski, 1998). The laser energy of the "green MLA" was again chosen to provide a balanced transponder link with SLR2000 assuming the $25 \mathrm{~cm}$ MLA receive aperture. The instrument characteristics are summarized in Table 3.

In this low SNR example, we select a range bin size, $\tau_{\mathrm{b}}=2 \mathrm{~ns}$, which comfortably accommodates all of the signal photons in a single frame. We again assume the worst case scenario of totally unsynchronized clocks at Terminals A and B so that the receiver is initially ungated for the entire interpulse period of $0.5 \mathrm{~ms}$, but the higher repetition rate results in a much smaller number of range bins, i.e. $N_{\text {bin }}=2.5 \times 10^{5}$. We plot, in Fig. 11: (a) the optimum threshold; (b) the differential cell count; (c) the probability of detecting the signal cell; (d) the mean false alarms per laser fire; and (e) the number of interplanetary range measurements per second for the Earth and Mars terminals as a function of the synodic day for both night and day operations. Because we have assumed a "balanced" system, the frequency of recorded ranges is the same at both terminals.

The behavior of the high and low SNR links as a function of synodic phase are both qualitatively and quantitatively different. Instead of decreasing during the first quarter of the synodic cycle and leveling off as in the high SNR case, the optimum frame threshold in the photoncounting example climbs, under local daylight conditions, from roughly 10 pe to perhaps 70 pe for the Mars terminal and from 45 to 170 pe for the Earth terminal at maximum interplanetary range and then falls off symmetrically during the second half of the synodic cycle. This behavior occurs because, at longer interplanetary ranges, it takes longer to collect the signal photons necessary to unambiguously distinguish the signal cell from the noise cells. The longer frame time results in a higher mean noise count per cell, which in turn drives the optimum frame threshold higher. The effectiveness of our optimum threshold algorithm is demonstrated by the fact that the Normalized Differential Cell Count, with a maximum value of 1, never drops below 0.997 at either terminal during either day or night operations. Similarly, the probability of correctly identifying the signal cell is greater than $99.7 \%$ under all conditions, and the mean false alarm rate is less than one cell in 10,000 frames under the worst case condition of unsynchronized clocks. As in the high SNR case, the number of false alarms is driven down further once the opposite terminal is acquired and the range gate shrunk accordingly. We also note that the rate of range returns at maximum range is $4 \mathrm{~Hz}$, only slightly lower than the nearly fixed $5 \mathrm{~Hz}$ rate of the high SNR link, but rises to approximately $80 \mathrm{~Hz}$ near minimum range. It should be further noted that the Mixed Power-Aperture Product for this low SNR example is $0.013 \mathrm{Watt}-\mathrm{m}^{2}$ or 7.5 times smaller than the same product in the high SNR case $\left(.098 \mathrm{Watt}^{2} \mathrm{~m}^{2}\right)$. Thus, as postulated in Section 2.6, a significantly higher range sampling rate can be obtained with the low SNR photoncounting system over the full synodic period.

\section{Summary}

An Earth-Mars transponder link is within the SLR state-of-the-art. A NASA MOBLAS station is capable of ranging to a "green MOLA" over the full range of interplanetary distances at a $5 \mathrm{~Hz}$ rate using a conventional high SNR approach. In photon-counting mode, the automated SLR2000 system could range to an even smaller Messenger-sized transponder at Mars at a 
sampling rate of 4-80 Hz, depending on the interplanetary range. Analysis indicates that both transponders would be capable of independently acquiring the incoming signal and operating from the planetary surface under worst-case conditions of unsynchronized clocks and maximum solar background. These measurements would allow the generation of a precise time series of range and time offset between the ground and spaceborne clocks.

Because interplanetary light travel times are several orders of magnitude longer than is typical for artificial satellites, the absolute accuracy of the range and clock offset measurements is dominated by the frequency accuracy and stability of the ground and spaceborne clocks. Errors in the range vernier or atmospheric propagation delays are typically at the sub-centimeter level as for artificial satellite ranging. With a high quality atomic clock onboard, decimeter ranging and subnanosecond time transfers should be readily achievable. Further investigations are warranted to determine whether or not the spaceborne clock can be sufficiently "disciplined" by an Earth-based maser, via the time transfer process, to achieve centimeter or better ranging between the planets.

Signal acquisition of the opposite terminal can be accomplished in a two stage process under both local day and night conditions in the presence of a scattering atmosphere. The partially illuminated planetary disk has sufficient contrast against the local solar background to be seen in the CCD for the initial angular acquisition. Signal acquisition in the third dimension, or range, is simplified by the fact that the range rate can be predicted with an accuracy better than $1 \mathrm{~cm} / \mathrm{s}$. Nevertheless, wide range gates may be required during initial acquisition due to the following:

- A priori uncertainties in Earth-Mars range may be as large as $60 \mathrm{~km}$ based on comparison of recent generation JPL ephemerides

- There is uncertainty in the time of laser fire at the opposite terminal due to lack of synchronization in the two clocks as well as laser pulse jitter within the clock cycle whereas pulse jitter cancels out in single-ended SLR systems.

- The worst case uncertainty in pulse time of arrival is equal to the laser fire interval in the case of totally unsynchronized clocks firing at the same nominal rate.

Potential science benefits of laser transponders would include more precise ephemerides of selected planets and asteroids, improved knowledge of planetary librations and their interior makeup, the ability to perform more precise general relativistic experiments on an interplanetary scale, the study of solar gravity and internal mass distribution using Sun-orbiting satellites (much as we currently measure the Earth's gravity field using SLR to artificial satellites), and measurement of the mass distribution within the asteroid belt. The latter could be accomplished by landing a transponder on an asteroid and measuring the range variations as it circulates through the belt. Besides providing relief to an already overworked Deep Space Network (DSN) and an independent assessment of range and range-rate, engineering benefits of transponders might improve the reliability of interplanetary spacecraft operations through more accurate spacecraft trajectories and the ability to transfer time from Earth standards with subnanosecond accuracy.

Field tests of the SLR2000 system prototype are expected to be completed in 2002. The effectiveness of our signal extraction algorithms based on Post Detection Poisson Filtering have already been demonstrated in an airborne photon counting altimeter (Degnan, 2002). Ongoing laboratory transponder tests and simulations (Degnan et al., 1998), combined with continued launches of laser-based instrumentation such as the Geoscience Laser Altimer System (GLAS), 
Messenger, etc., should remove much of the technical risk and uncertainty in proposing an interplanetary laser transponder mission to fly within the next few years. The combined technical and scientific results from these varied programs should greatly enhance the credibility and scientific desirability of dual-purpose instrumentation like the combined Microlaser Altimeter Transponder (MAT) described in Fig. 7.

\section{Acknowledgements}

The author wishes to acknowledge the following colleagues at the NASA Goddard Space Flight Center who have contributed to the development of the transponder mission scenario and instrument concept: Jan McGarry, Phillip Dabney, Thomas Zagwodzki, Michael Tierney, and a NASA Space Academy student, Matthew Weatherly. Dr. Frank Lemoine of NASA/GSFC provided estimates of the range and range-rate uncertainties in the Earth to Mars link based on different versions of planetary ephemerides provided by the Jet Propulsion Laboratory (JPL). Jon Giorgio of JPL provided similar estimates for a link between Earth and the asteroid Vesta. This paper has also benefited from discussions with Prof. Ken Nordtvedt, University of Montana, and Dr. David E. Smith, NASA/GSFC, on the potential scientific applications of interplanetary transponders and with Dr. Peter J. Shelus, University of Texas Center for Space Research at Austin, on lunar laser ranging issues and procedures.

\section{Appendix A. Noise models}

\section{A1. Solar and lunar scattering in the local atmosphere}

Since the effective thickness of the atmosphere, $h_{\mathrm{a}}(\sim 20 \mathrm{~km}$ for Earth) is small relative to the volumetric mean radius of the planet, $R_{\mathrm{B}}(6378 \mathrm{~km}$ for Earth), we can accurately represent the atmosphere (except very near the horizon) by a stratified rectangular slab with the station centered on the bottom face. In our model, the atmospheric extinction coefficient, $\mu(z)$, will be assumed to be a function of the vertical height only, i.e. no horizontal gradients are assumed. The atmospheric transmission at zenith is then given by

$$
T_{\mathrm{B}}=T_{\mathrm{B}}\left(h_{s}^{\mathrm{B}}, \infty\right)=\exp \left[-\int_{h_{s}^{\mathrm{B}}}^{\infty} \mathrm{d} z^{\prime} \mu_{\mathrm{B}}\left(z^{\prime}\right)\right]
$$

where $h_{s}^{\mathrm{B}}$ is the height of the $\mathrm{B}$ terminal station above some reference height (e.g. sea level on Earth).

The solar irradiance can be viewed as a plane wave incident on the top plane of the atmosphere at a solar zenith angle, $\theta_{\mathrm{S}}$, as viewed from the terminal on Planet $\mathrm{B}$. If we ignore the slight bending of the solar rays as they propagate through the vertically stratified atmosphere, the transmitted irradiance to a differential volume at an arbitrary height above sea level, $z$, is given by 


$$
N_{\lambda}^{\mathrm{B}} \exp \left(-\sec \theta_{S} \int_{z}^{\infty} \mathrm{d} z^{\prime} \mu_{\mathrm{B}}\left(z^{\prime}\right)\right)=N_{\lambda}^{\mathrm{B}}\left[T_{\mathrm{B}}(z, \infty)\right]^{\sec \theta_{\mathrm{S}}}
$$

where $N_{\lambda}^{\mathrm{B}}$ is the exoatmospheric solar irradiance at Planet B. At the $532 \mathrm{~nm}$ wavelength, the solar irradiance at Earth has a value $0.2 \mathrm{Watts} / \mathrm{m}^{2}-\mathrm{A}^{\circ}$ and falls off as $1 / R_{\mathrm{B}}^{2}$ where $R_{\mathrm{B}}$ is the distance of Planet B from the sun expressed in Astronomical Units (1 AU $\sim 150$ million $\mathrm{km}$ ).

The receiver has a solid angle field of view (FOV) given by

$$
\Omega_{r}^{\mathrm{B}}=\pi \theta_{r \mathrm{~B}}^{2}
$$

where $\theta_{r \mathrm{~B}}$ is the half angle of the far field cone viewed by the receiver. When the opposite terminal is at a zenith angle $\theta_{\mathrm{B}}$, the scattered solar power collected locally by a receiver with collecting aperture $A_{\mathrm{B}}$ and spectral bandwidth $\Delta \lambda_{\mathrm{B}}$ is given by the following integral

$$
\begin{aligned}
P_{\mathrm{ls}}^{\mathrm{B}} & =\int_{0}^{\infty} \mathrm{d} s N_{\lambda}^{\mathrm{B}}\left(\Delta \lambda_{\mathrm{B}}\right) \frac{A_{\mathrm{B}}}{s^{2}}\left[T\left(h_{s}^{\mathrm{B}}, z\right)\right]^{\sec \theta_{\mathrm{B}}} \frac{\beta_{\mathrm{B}}(z)}{4 \pi}\left[\Omega_{r}^{\mathrm{B}} s^{2}\right]\left[T_{\mathrm{B}}(z, \infty)\right]^{\sec \theta_{\mathrm{S}}} \\
& =\frac{N_{\lambda}^{\mathrm{B}}\left(\Delta \lambda_{\mathrm{B}}\right) A_{\mathrm{B}} \Omega_{r}^{\mathrm{B}} \sec \theta_{\mathrm{B}}}{4 \pi} \int_{h_{s}^{\mathrm{B}}}^{\infty} \mathrm{d} z \beta_{\mathrm{B}}(z)\left[T_{\mathrm{B}}\left(h_{s}^{\mathrm{B}}, z\right)\right]^{\sec \theta_{\mathrm{B}}}\left[T_{\mathrm{B}}(z, \infty)\right]^{\sec \theta_{\mathrm{S}}}
\end{aligned}
$$

where

$$
s=\left(z-h_{s}^{\mathrm{B}}\right) \sec \theta_{\mathrm{B}}
$$

is the range to the differential volume, $\beta_{\mathrm{B}}(z)$ is the volumetric scattering coefficient at a height $z$ above sea level, and we have assumed isotropic scattering. For wavelengths far from absorption lines, the atmospheric extinction is due entirely to scattering so we can set $\beta_{\mathrm{B}}(z)=\mu_{\mathrm{B}}(z)$. Using (A1) and (A4), the rate at which solar background light is converted into photoelectrons by receiver $\mathrm{B}$ is

$$
\begin{aligned}
\stackrel{\bullet}{n_{\mathrm{s}}^{\mathrm{B}}} & =\frac{\eta_{q}^{\mathrm{B}} \eta_{r}^{\mathrm{B}}}{h v} \frac{N_{\lambda}^{\mathrm{B}}\left(\Delta \lambda_{\mathrm{B}}\right) A_{\mathrm{B}} \Omega_{r}^{\mathrm{B}} \sec \theta_{\mathrm{B}}}{4 \pi} \int_{h_{s}^{\mathrm{B}}}^{\infty} \mathrm{d} z \beta_{\mathrm{B}}(z)\left[T_{\mathrm{B}}\left(h_{s}^{\mathrm{B}}, z\right)\right]^{\sec \theta_{\mathrm{B}}}\left[T_{\mathrm{B}}(z, \infty)\right]^{\sec \theta_{\mathrm{S}}} \\
& =\frac{\eta_{q}^{\mathrm{B}} \eta_{r}^{\mathrm{B}}}{h v} \frac{N_{\lambda}^{\mathrm{B}}\left(\Delta \lambda_{\mathrm{B}}\right) A_{\mathrm{B}} \Omega_{r}^{\mathrm{B}} \sec \theta_{\mathrm{B}}}{4 \pi} \int_{h_{s}^{\mathrm{B}}}^{\infty} \mathrm{d} z \mu_{\mathrm{B}}(z) \exp \left[-\sec \theta_{\mathrm{B}} \int_{h_{s}^{\mathrm{B}}}^{z} \mathrm{~d} z^{\prime} \mu_{\mathrm{B}}\left(z^{\prime}\right)-\sec \theta_{\mathrm{S}} \int_{z}^{\infty} \mathrm{d} z^{\prime} \mu_{\mathrm{B}}\left(z^{\prime}\right)\right]
\end{aligned}
$$

where $\eta_{q}^{\mathrm{B}}$ is the detector quantum efficiency, $\eta_{r}^{\mathrm{B}}$ is the receiver optical throughput efficiency, and $h v$ is the photon energy at the laser wavelength. In order to evaluate the final integral in (A5), we define the new variable

$$
\xi=\sec \theta_{\mathrm{B}} \int_{h_{s}^{\mathrm{B}}}^{z} \mathrm{~d} z^{\prime} \mu_{\mathrm{B}}\left(z^{\prime}\right)+\sec \theta_{\mathrm{S}} \int_{z}^{\infty} \mathrm{d} z^{\prime} \mu_{\mathrm{B}}\left(z^{\prime}\right)
$$


which further implies

$$
\mathrm{d} \xi=\mathrm{d} z \mu_{\mathrm{B}}(z)\left[\sec \theta_{\mathrm{B}}-\sec \theta_{\mathrm{S}}\right]
$$

Substituting (A6) into (A5) and evaluating the trivial integral yields

$$
\stackrel{\bullet}{n_{\mathrm{ls}}^{\mathrm{B}}}=\frac{\eta_{q}^{\mathrm{B}} \eta_{r}^{\mathrm{B}}}{h v} \frac{N_{\lambda}^{\mathrm{B}}\left(\Delta \lambda_{\mathrm{B}}\right) A_{\mathrm{B}} \Omega_{r}^{\mathrm{B}}}{4 \pi}\left\{\sec \theta_{\mathrm{B}} T_{\mathrm{B}}^{\mathrm{sec} \theta_{\mathrm{B}}}\left[\frac{1-T_{\mathrm{B}}^{\sec \theta_{\mathrm{s}}-\sec \theta_{\mathrm{B}}}}{\sec \theta_{\mathrm{S}}-\sec \theta_{\mathrm{B}}}\right]\right\} .
$$

It should be pointed out that the atmospheric noise model makes no assumptions regarding the distribution of scatterers with altitude. The model does assume no horizontal gradients, however, and therefore depends only on the zenith transmission, $T_{\mathrm{B}}$, between the station and the "top" of the planetary atmosphere and the local zenith angles of the Sun, $\theta_{\mathbf{S}}$, and of the opposite terminal, $\theta_{\mathrm{B}}$. Note also that as $T_{\mathrm{B}} \rightarrow 1$ (no scattering), the background count rate due to solar scatter off the atmosphere correctly goes to zero for all values of $\theta_{\mathbf{S}}$ and $\theta_{\mathbf{B}}$.

When the solar and spacecraft zenith angles are equal $\left(\theta_{\mathbf{S}}=\theta_{\mathbf{B}}\right)$, the bracketed [] term in (A7) becomes indeterminate, but we can use L'Hospital's rule to obtain

$$
\lim _{\omega \rightarrow 0} \frac{1-T_{\mathrm{B}}^{\omega}}{\omega}=\ln \left(\frac{1}{T_{\mathrm{B}}}\right)
$$

for the special case when $\omega=\sec \theta_{\mathrm{S}}-\sec \theta_{\mathbf{B}}=0$. This approximation also holds in the limit of a highly transmissive atmosphere for arbitrary $\theta_{\mathrm{S}}$ and $\theta_{\mathrm{B}}$ yielding an approximate expression

$$
\stackrel{\bullet}{n_{\mathrm{ls}}^{\mathrm{B}}} \simeq \frac{\eta_{q}^{\mathrm{B}} \eta_{r}^{\mathrm{B}}}{h v} \frac{N_{\lambda}^{\mathrm{B}}\left(\Delta \lambda_{\mathrm{B}}\right) A_{\mathrm{B}} \Omega_{r}^{\mathrm{B}}}{4 \pi}\left\{T_{\mathrm{B}}^{\mathrm{sec} \theta_{\mathrm{B}}} \ln T_{\mathrm{B}}^{\sec \theta_{\mathrm{B}}}\right\}
$$

which is independent of the solar zenith angle. In the case of a planetary lander communicating with an Earth station, (A7) can clearly be used to assess noise at the opposite terminal by interchanging the superscripts and subscripts, A and B.

Plots of (A7) and (A9) show the following general results:

- For all values of the spacecraft zenith angle, $\theta_{\mathrm{B}}$, or local zenith (i.e. directly above the station) transmission, $T_{\mathrm{B}}$, the noise rate due to local atmospheric scattering of solar radiation is always greatest when the Sun is directly overhead $\left(\theta_{\mathrm{s}}=0\right)$ and decreases monotonically to zero as the Sun drops below the horizon.

- For higher values of the local zenith transmission and all solar zenith angles, the noise count rate grows from a minimum at local zenith $\left(\theta_{\mathrm{B}}=0\right)$ and reaches a maximum at very high spacecraft zenith angles $\left(\theta_{\mathrm{B}}>80^{\circ}\right)$. This is illustrated in Fig. 4 where we have chosen $T_{\mathrm{B}}=0.7$, corresponding to a standard clear Earth atmosphere at $532 \mathrm{~nm}$. For very high atmospheric transmissions approaching unity, the noise count rate at most spacecraft zenith angles approaches zero because of the low density of scatterers contained within the viewed atmospheric volume. An exception is very near the horizon where the long atmospheric path length and correspondingly larger viewed volume compensates for the low density of scatterers. 
- For low values of the zenith transmission (not shown in Fig. 4) and all solar zenith angles, the noise count rate drops off monotonically from a peak at local zenith $\left(\theta_{\mathbf{B}}=0\right)$. This is due to the poor penetration of solar radiation at high zenith angles.

- As demonstrated in Fig. 4, the approximate expression in (A9) gives fairly good results for even moderate atmospheric transmissions $\left(T_{\mathrm{B}} \sim 0.7\right)$ for spacecraft zenith angles below about 60 degrees. The agreement of (A9) with the exact result (A7) improves for higher atmospheric transmissions.

At full Moon, the lunar spectral irradiance outside the atmosphere has the value $N_{\lambda}^{\mathrm{M}}=4.8 \times 10^{-7} \mathrm{~W} / \mathrm{m}^{2}-\mathrm{A}^{\circ}$ at a wavelength of $532 \mathrm{~nm}$ (Zissis, 1993), or over six orders of magnitude less than the solar value, which can be substituted into the preceding equations to determine the worst case reflected lunar light scattering from the atmosphere.

\section{A2. Planetary albedo}

We can use the results and techniques of Appendix A1 to develop a model for the photoelectron count rate resulting from solar scattering from the viewed planet (planetary albedo). We propose to use these photoelectrons in the initial acquisition of the opposite terminal by imaging the host planet onto a pixellated imaging detector such as a CCD array. In the ranging portion of the receiver, these count rates also represent an inescapable source of background noise, which the laser signal from the opposite terminal must overcome.

The background rate from planetary albedo can consist of two components - surface and atmospheric (if any) scattering. The worst case background occurs when the two communicating planets and the Sun fall on or near a linear configuration, which also coincides with the points of minimum and maximum interplanetary range (see Section 5). Near the point of closest approach, the terminal on the innermost planet is in darkness and ranging to the terminal on the outer planet which is bathed in daylight and cannot see the inner planet against the blinding background of the Sun. Near maximum range, the two terminals are both in daylight and the transponder link is blocked by the Sun itself. Thus, transponder operations can only occur when the aspect angles, $\alpha_{\mathrm{A}}$ and $\alpha_{\mathrm{B}}$ (see Fig. 3a), are sufficiently far from the values 0 or $180^{\circ}$ to avoid direct observation of the Sun by one or both terminals. The fractional disk area illuminated by the Sun drops monotonically from 1 to 0 as the aspect angle varies between 0 and $180^{\circ}$ as shown in Fig. 3b. The fractional disk area viewed by transponders on Earth and Mars are plotted as a function of synodic phase in Fig. 3c.

In modelling planetary albedo contributions to transponder noise, we assume a spherical planet surrounded by a relatively thin atmospheric shell. The noise count rate in receiver B due to solar radiation scattered from the surface of Planet $\mathrm{A}$ is given by the equation

$$
\stackrel{\bullet}{n_{\mathrm{ps}}^{\mathrm{B}}}=\frac{\eta_{q}^{\mathrm{B}} \eta_{r}^{\mathrm{B}}}{h v} \frac{N_{\lambda}^{\mathrm{A}}\left(\Delta \lambda_{\mathrm{B}}\right) A_{\mathrm{B}} T_{\mathrm{B}}^{\sec \left(\theta_{\mathrm{B}}\right)} r_{\mathrm{A}}^{2}}{\pi R^{2}}\left[\int \mathrm{d} \varphi \int \mathrm{d} \theta \rho_{\mathrm{A}}(\theta, \varphi) \sin \theta \cos \theta T_{\mathrm{A}}^{\sec \theta+\sec \gamma}\right]
$$

where we have assumed Lambertian scattering from the surface and included the effects of the atmospheric transmission at both planets but ignored the slight geometric bending of the solar rays in the atmosphere. In $(22), \rho_{\mathrm{A}}(\theta, \varphi)$ is the reflectance of Planet A's surface at the operating 
wavelength, $r_{A}$ is the volumetric mean radius of Planet $\mathrm{A}, \theta_{\mathbf{B}}$ is the local zenith angle of Terminal A as viewed from Terminal B,

$$
\sec \gamma=\left[\sin \theta \sin \alpha_{\mathrm{B}} \cos \varphi+\cos \theta \cos \alpha_{\mathrm{B}}\right]^{-1}
$$

and $\alpha_{\mathrm{B}}$ is the aspect angle, defined as the angle subtended by two vectors, both originating from Planet B, directed toward the Sun and Planet A respectively (see Fig. 3). The aspect angle of the solar-illuminated surface of Planet B (Mars), as viewed from Planet A (Earth), is given mathematically by

$$
\alpha_{\mathrm{A}}(t)=\cos ^{-1}\left[-\frac{\vec{R}_{\mathrm{A}}(t)}{R_{\mathrm{A}}(t)} \bullet \frac{\vec{R}(t)}{R(t)}\right]
$$

where $\vec{R}(t)=\overrightarrow{R_{\mathrm{B}}}(t)-\overrightarrow{R_{\mathrm{A}}}(t)$ is the instantaneous interplanetary vector. Similarly, the aspect angle of the solar-illuminated surface of Planet A, as viewed from Planet B, is

$$
\alpha_{\mathrm{B}}(t)=\cos ^{-1}\left[\frac{\vec{R}_{\mathrm{B}}(t)}{R_{\mathrm{B}}(t)} \bullet \frac{\vec{R}(t)}{R(t)}\right]
$$

The two-dimensional integral in (A11a) is over the solar-illuminated surface area within the receiver FOV. If Planet $\mathrm{A}$ is sufficiently far away so that it is contained totally within the Terminal B FOV, the integral is over the area where the hemispheres in the direction of the two aforementioned vectors overlap. If the opposite planet is contained entirely within the receiver FOV and the atmosphere is either highly transmissive or non-existent $\left(T_{\mathrm{A}} \cong 1\right)$, (A11a) can be approximated by

$$
\stackrel{\bullet}{n_{\mathrm{ps}}^{\mathrm{B}}}=\frac{\eta_{q}^{\mathrm{B}} \eta_{r}^{\mathrm{B}}}{h v} \frac{N_{\lambda}^{\mathrm{A}}\left(\Delta \lambda_{\mathrm{B}}\right) A_{\mathrm{B}} \rho_{\mathrm{A}} T_{\mathrm{B}}^{\sec \left(\theta_{\mathrm{B}}\right)} r_{\mathrm{A}}^{2}}{R^{2}} g_{\mathrm{ps}}\left(\alpha_{\mathrm{A}}\right)
$$

where the geometric factor

$$
\begin{aligned}
& g_{\mathrm{ps}}\left(0 \leqslant \alpha_{\mathrm{A}} \leqslant \frac{\pi}{2}\right)=\cos ^{2} \alpha_{\mathrm{A}}+\frac{2}{\pi} \int_{\cos \alpha_{\mathrm{A}}}^{1} x \cos ^{-1}\left(-\cot \alpha_{\mathrm{A}}\left(\frac{\sqrt{1-x^{2}}}{x}\right)\right) \mathrm{d} x \\
& g_{\mathrm{ps}}\left(\frac{\pi}{2} \leqslant \alpha_{\mathrm{A}} \leqslant \pi\right)=\frac{2}{\pi} \int_{\left|\cos \alpha_{\mathrm{A}}\right|}^{1} x \cos ^{-1}\left(-\cot \alpha_{\mathrm{A}}\left(\frac{\sqrt{1-x^{2}}}{x}\right)\right) \mathrm{d} x
\end{aligned}
$$

has a value between $0\left(\alpha_{\mathrm{A}}=\pi\right)$ and $1\left(\alpha_{\mathrm{A}}=0\right)$, and, since the planets spin on their axis, we have substituted a globally-averaged value for the surface reflectance, $\rho_{\mathrm{A}}$. 
Following an analysis similar to that in Appendix A1, the noise count rate in receiver B due to solar power scattered from the atmosphere of Planet A can be shown to be

$$
\begin{aligned}
n_{\mathrm{pa}}^{\mathrm{B}} & =\frac{\eta_{q}^{\mathrm{B}} \eta_{r}^{\mathrm{B}}}{h v} \frac{N_{\lambda}^{\mathrm{A}} \Delta \lambda_{\mathrm{B}} A_{\mathrm{B}} T_{\mathrm{B}}^{\mathrm{sec}\left(\theta_{\mathrm{B}}\right)}}{4 \pi R^{2}}\left[\int_{R_{\mathrm{A}}}^{r_{\mathrm{A}}+h_{\mathrm{A}}} \mathrm{d} r r^{2} \beta(r) \int \mathrm{d} \varphi \int \mathrm{d} \theta \sin \theta T_{\mathrm{A}}^{\sec \theta+\sec \gamma}\right] \\
& =\frac{\eta_{q}^{\mathrm{B}} \eta_{r}^{\mathrm{B}}}{h v} \frac{N_{\lambda}^{\mathrm{A}} \Delta \lambda_{\mathrm{B}} A_{\mathrm{B}} T_{\mathrm{B}}^{\sec \left(\theta_{\mathrm{B}}\right)}}{4 \pi R^{2}}\left\{\int \mathrm{d} \varphi \int \mathrm{d} \theta \sin \theta \int_{r_{\mathrm{A}}}^{r_{\mathrm{A}}+h_{\mathrm{A}}} \mathrm{d} r r^{2} \mu(r) \exp \left[-[\sec \theta+\sec \gamma] \int_{r}^{\infty} \mathrm{d} r^{\prime} \mu\left(r^{\prime}\right)\right]\right\}
\end{aligned}
$$

where $h_{\mathrm{A}}$ is the height of the atmosphere above Planet A's surface. In (A13), we have used the spherical coordinate versions of (A1) and (A2) and again assumed that there is little or no atmospheric absorption at the laser wavelength and that the volumetric scattering coefficient, $\beta$, can be replaced by the volumetric extinction coefficient, $\mu$. In our thin atmosphere approximation, we have $h_{\mathrm{A}} \ll r_{\mathrm{A}}$ and can therefore approximate $r^{2}$ by $r_{\mathrm{A}}^{2}$ in the last integral in (A13). Using (A6), we can then evaluate the radial integral to obtain

$$
\stackrel{\bullet}{n_{\mathrm{pa}}^{\mathrm{B}}}=\frac{\eta_{q}^{\mathrm{B}} \eta_{r}^{\mathrm{B}}}{h v} \frac{N_{\lambda}^{\mathrm{A}} \Delta \lambda_{\mathrm{B}} A_{\mathrm{B}} r_{\mathrm{A}}^{2} T_{\mathrm{B}}^{\sec \theta_{\mathrm{B}}}}{4 \pi R^{2}}\left\{\int \mathrm{d} \varphi \int \mathrm{d} \theta \sin \theta \frac{1-\exp \left[(\sec \theta+\sec \gamma) \ln \left(T_{\mathrm{A}}\right)\right]}{\sec \theta+\sec \gamma}\right\}
$$

which, in the absence of a scattering atmosphere on Planet $\mathrm{A}\left(T_{\mathrm{A}}=1\right)$, correctly gives zero for the atmospheric contribution to the background. For a highly transmissive atmosphere, we can approximate the exponential in (A14) by the first two terms in a Taylor expansion, which yields the approximate expression

$$
\stackrel{\bullet}{n_{\mathrm{pa}}^{\mathrm{B}}} \cong \frac{\eta_{q}^{\mathrm{B}} \eta_{r}^{\mathrm{B}}}{h v} \frac{N_{\lambda}^{\mathrm{A}} \Delta \lambda_{\mathrm{B}} A_{\mathrm{B}} r_{\mathrm{A}}^{2} T_{\mathrm{B}}^{\mathrm{sec} \theta_{\mathrm{B}}}}{2 R^{2}} \ln \left(\frac{1}{T_{\mathrm{A}}}\right) g_{\mathrm{as}}\left(\alpha_{\mathrm{A}}\right)
$$

where the geometric factor for atmospheric scattering,

$$
\begin{aligned}
& g_{\text {as }}\left(0 \leqslant \alpha_{\mathrm{A}} \leqslant \frac{\pi}{2}\right)=1-\sin \alpha_{\mathrm{A}}+\frac{1}{\pi} \int_{0}^{\sin \alpha_{\mathrm{A}}} \cos ^{-1}\left(-\cot \alpha_{\mathrm{A}} \frac{x}{\sqrt{1-x^{2}}}\right) \mathrm{d} x \\
& g_{\text {as }}\left(\frac{\pi}{2} \leqslant \alpha_{\mathrm{A}} \leqslant \pi\right)=\frac{1}{\pi} \int_{0}^{\sin \alpha_{\mathrm{A}}} \cos ^{-1}\left(-\cot \alpha_{\mathrm{A}} \frac{x}{\sqrt{1-x^{2}}}\right) \mathrm{d} x
\end{aligned}
$$

again takes on values between $0\left(\alpha_{\mathrm{A}}=\pi\right)$ and $1\left(\alpha_{\mathrm{A}}=0\right)$.

Summing (A12a) and (A15a) gives an approximate expression for the noise count rate for the total planetary albedo, i.e.

$$
\stackrel{\bullet}{n_{\mathrm{sa}}^{\mathrm{B}}} \cong \frac{\eta_{q}^{\mathrm{B}} \eta_{r}^{\mathrm{B}}}{h v} \frac{N_{\lambda}^{\mathrm{A}} \Delta \lambda_{\mathrm{B}} A_{\mathrm{B}} r_{\mathrm{A}}^{2} T_{\mathrm{B}}^{\mathrm{sec} \theta_{\mathrm{B}}}}{R^{2}}\left[\rho_{\mathrm{A}} g_{\mathrm{ps}}^{\mathrm{B}}\left(\alpha_{\mathrm{A}}\right)+\frac{1}{2} \ln \left(\frac{1}{T_{\mathrm{A}}}\right) g_{\mathrm{as}}\left(\alpha_{\mathrm{A}}\right)\right]
$$

where the subscript sa refers to surface and atmosphere. 


\section{A3. Laser backscatter from the local atmosphere}

Unlike the sources of noise discussed previously, which are all constant in time, the contribution of laser backscattering to the overall noise count falls rapidly with the time after pulse emission, $\tau$. At large spacecraft zenith angles $\left(\theta_{\mathrm{B}} \sim 70^{\circ}\right)$, the effects of laser backscatter at the Earth station can be significant for time scales up to $100 \mu \mathrm{s}$, which corresponds roughly to the time it takes laser photons to reach the uppermost layer of the atmosphere and return at low elevation angles. Thus, photons arriving from the opposite terminal during this early segment of the local laser fire interval will be competing with an additional time-dependent noise source. The effects of laser backscattering can often be suppressed in ranging systems by employing a bistatic design, i.e. one having truly separate transmit and receive optics with parallel but not coaxial paths. Where common telescope optics are used for the transmit and receive beams, an alternative approach is to spatially isolate the transmit and receive beams through a combination of "aperture sharing" and aggressive stray light suppression. Bistatic receivers have the additional virtue that "transmitter point-ahead" can be more easily accommodated (see Section 4.2).

For the sake of completeness, the backscattered laser power collected by a coaxial, monostatic receiver $\mathrm{B}$ is given by the lidar equation

$$
P_{\mathrm{bs}}^{\mathrm{B}}(s)=\left(\frac{E_{\mathrm{B}} c}{2}\right) \frac{\beta_{\mathrm{B}}(s)}{4 \pi} \frac{A_{\mathrm{B}}}{s^{2}} \exp \left[-2 \int_{0}^{s} \mathrm{~d} s^{\prime} \mu_{\mathrm{B}}\left(s^{\prime}\right)\right]
$$

where $s$ is again the range to the scattering volume, $c$ is the speed of light, and $E_{\mathrm{B}}$ is the transmitted laser energy. For the stratified and non-absorbing atmosphere considered previously, the rate of back-scattered photoelectron generation at the detector is equal to

$$
\stackrel{\bullet}{n_{\mathrm{bs}}^{\mathrm{B}}}=\frac{\eta_{q}^{\mathrm{B}} \eta_{r}^{\mathrm{B}}}{h v} P_{\mathrm{bs}}^{\mathrm{B}}(s)=\left(\frac{E_{\mathrm{B}} c}{2}\right) \frac{\eta_{q}^{\mathrm{B}} \eta_{r}^{\mathrm{B}}}{h v} \frac{\mu_{\mathrm{B}}(z)}{4 \pi} \frac{A_{\mathrm{B}}}{\left[\left(z-h_{\mathrm{s}}^{\mathrm{B}}\right) \sec \theta_{\mathrm{B}}\right]^{2}} \exp \left[-2 \sec \theta_{\mathrm{B}} \int_{h_{s}}^{z} \mathrm{~d} z^{\prime} \mu_{\mathrm{B}}\left(z^{\prime}\right)\right]
$$

Experimentally, one finds the following height dependence for the extinction coefficient (RCA, 1968)

$$
\mu_{\mathrm{B}}(z)=\mu_{\mathrm{B}}^{0} \exp \left(-z / h_{\mathrm{sc}}^{\mathrm{B}}\right)
$$

where $\mu_{\mathrm{B}}^{0}$ is the extinction coefficient at the nominal lowest-lying surface (sea level on Earth) and $h_{\mathrm{sc}}^{\mathrm{B}}$ is the atmospheric scale height for Planet B. Substituting the latter expression into (13) yields the relation

$$
T_{\mathrm{B}}=T_{\mathrm{B}}\left(h_{\mathrm{s}}^{\mathrm{B}}, \infty\right)=\exp \left[-\mu_{\mathrm{B}}^{0} h_{\mathrm{sc}}^{\mathrm{B}} \mathrm{e}^{-\frac{h_{\mathrm{s}}^{\mathrm{B}}}{h_{\mathrm{Bc}}^{\mathrm{B}}}}\right]
$$

Using (A19) and (A20) in (A18), we can write 


$$
\stackrel{\bullet}{n_{\mathrm{bs}}^{\mathrm{B}}}=\left(\frac{E_{\mathrm{B}} A_{\mathrm{B}} c}{8 \pi h_{\mathrm{sc}}^{\mathrm{B}}}\right) \frac{\eta_{q}^{\mathrm{B}} \eta_{r}^{\mathrm{B}}}{h v} \frac{\exp \left(-\frac{z-h_{s}^{\mathrm{B}}}{h_{\mathrm{sc}}^{\mathrm{B}}}\right)}{\left[\left(z-h_{s}^{\mathrm{B}}\right) \sec \theta_{\mathrm{B}}\right]^{2}} \ln \left(\frac{1}{T_{\mathrm{B}}}\right) T_{\mathrm{B}}^{2 \sec \theta_{\mathrm{B}}}\left[1-\exp \left(-\frac{\left(z-h_{s}^{\mathrm{B}}\right)}{h_{\mathrm{sc}}^{\mathrm{B}}}\right)\right]
$$

We can now choose to express (A21) as a function of the time from laser fire, i.e.

$$
\tau=\frac{2 s}{c}=\frac{2 \sec \theta_{\mathrm{B}}}{c}\left(z-h_{s}^{\mathrm{B}}\right)
$$

to obtain our final result

$$
n_{\mathrm{bs}}^{\mathrm{B}}(\tau)=\frac{\eta_{q}^{\mathrm{B}} \eta_{r}^{\mathrm{B}} A_{\mathrm{B}}}{2 \pi h v} \frac{E_{\mathrm{B}}}{h_{\mathrm{sc}}^{\mathrm{B}} c}\left[\ln \left(\frac{1}{T_{\mathrm{B}}}\right) T_{\mathrm{B}}^{2 \sec \theta_{\mathrm{B}}}\left[1-\exp \left(-\frac{c \tau}{2 h_{\mathrm{sc}}^{\mathrm{B}} \sec \theta_{\mathrm{B}}}\right)\right]\right]\left[\frac{\mathrm{e}^{-\frac{c \tau}{2 h_{\mathrm{sc}}^{\mathrm{B}} \sec \theta_{\mathrm{B}}}}}{\tau^{2}}\right]
$$

which, due to the logarithmic term, also goes to zero in the absence of a scattering atmosphere.

\section{Appendix B. Transmitter point ahead}

The point-ahead angle for the transmitter at Terminal $\mathrm{A}$ is given by the equation

$$
\xi_{\mathrm{A}}(t)=\cos ^{-1}\left\{\frac{\overrightarrow{R_{\mathrm{B}}}\left(t+t_{f}^{\mathrm{A}}\right)-\overrightarrow{R_{\mathrm{A}}}(t)}{\left|\overrightarrow{R_{\mathrm{B}}}\left(t+t_{f}^{\mathrm{A}}\right)-\overrightarrow{R_{\mathrm{A}}}(t)\right|} \bullet \frac{\overrightarrow{R_{\mathrm{B}}}\left(t-t_{b}^{\mathrm{A}}\right)-\overrightarrow{R_{\mathrm{A}}}(t)}{\left|\overrightarrow{R_{\mathrm{B}}}\left(t-t_{b}^{\mathrm{A}}\right)-\overrightarrow{R_{\mathrm{A}}}(t)\right|}\right\}
$$

where $t_{f}^{\mathrm{A}}$ and $t_{b}^{\mathrm{A}}$ are the "forward" and "backward" one-way flight times of a photon leaving and arriving at Earth at a time $t$ respectively. In terms of general ephemerides, these photon flight times are the solutions to the equations

$$
c \tau_{f}^{\mathrm{A}}=\left|\overrightarrow{R_{\mathrm{B}}}\left(t+\tau_{f}^{\mathrm{A}}\right)+\overrightarrow{r_{\mathrm{B}}}\left(t+\tau_{f}^{\mathrm{A}}\right)-\overrightarrow{R_{\mathrm{A}}}(t)-\overrightarrow{r_{\mathrm{A}}}(t)\right|
$$

and

$$
c \tau_{b}^{\mathrm{A}}=\left|\overrightarrow{R_{\mathrm{B}}}\left(t-\tau_{b}^{\mathrm{A}}\right)+\overrightarrow{r_{\mathrm{B}}}\left(t-\tau_{b}^{\mathrm{A}}\right)-\overrightarrow{R_{\mathrm{A}}}(t)-\overrightarrow{r_{\mathrm{A}}}(t)\right|
$$

where, in computing the pulse time of flight, we have included both the planetary Center of Mass motions about the Sun as well as the terminal motions about the planetary axis of rotation as discussed in Section 4.3. The latter equations can be solved by substituting the instantaneous 
range at time $t$ divided by the speed of light into the RHS of (B2) and continuing the iteration until the desired accuracy is achieved.

For the purposes of calculating transmitter point-ahead, we can ignore planetary rotation and use the first order vector Taylor expansion

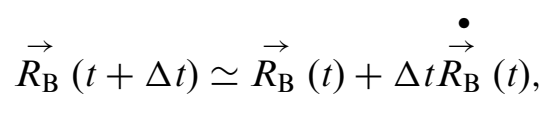

to obtain approximate expressions for the forward and backward photon times of flight between Earth and Mars, i.e.

$$
\begin{aligned}
& t_{f}^{\mathrm{A}}(t) \simeq \frac{R(t)}{c\left(1-\varepsilon_{\mathrm{A}}(t)\right)} \\
& t_{b}^{\mathrm{A}}(t) \simeq \frac{R(t)}{c\left(1+\varepsilon_{\mathrm{A}}(t)\right)}
\end{aligned}
$$

where the first order correction term

$$
\varepsilon_{\mathrm{A}}(t)=\frac{\vec{R}(t)}{R(t)} \bullet \frac{\stackrel{\overrightarrow{R_{\mathrm{B}}}}{c}(t)}{c}
$$

modifies the calculated photon flight time between Earth and Mars by less than one part in $10^{4}$. Eqs. (B4a)-(B4c) can now be used in (B1) to compute the transmitter point ahead angle at Terminal A. As always, the transmitter point ahead angle for Terminal B is obtained by interchanging the $\mathrm{A}$ and $\mathrm{B}$ superscripts/subscripts in the latter equations.

\section{References}

Abbott, R.I., Shelus, P.J., Mulholland, R., Silverberg, E., 1973. Laser observations of the Moon: identification and construction of normal points for 1969-1971. The Astronomical Journal 78, 784-793.

Bender, P.L., Faller, J.E., Hall, J.L., Degnan, J.J., Dickey, J.O., Newhall, X.X., Williams, J.G., King, R.W., Machnik, L.O., O’Gara, D., Ricklefs, R.L., Shelus, P.J., Whipple, A.L., Wiant, J.R., Veillet, C., 1990. Microwave and Optical Lunar Transponders, in Astrophysics from the Moon, AIP Conf. Proc. Series. American Institute of Physics, New York.

Degnan, J.J., 1985. Satellite laser ranging: current status and future prospects. IEEE Transactions on Geoscience and Remote Sensing GE-23, 398-413.

Degnan, J.J., 1993. Millimeter accuracy satellite laser ranging: a review. In: Smith, D.E., Turcotte, D.L. (Eds.), Contributions of Space Geodynamics: Technology. AGU Geodynamics Series, Vol. 25. pp. 133-162.

Degnan, J.J., 1996. Compact laser transponders for interplanetary ranging and time transfer. In: Proc. 10th International Workshop on Laser Ranging. Shanghai, China, 11-15 November, pp. 24-31.

Degnan, J.J., McGarry, J.F., 1997. SLR2000: eyesafe and autonomous single photoelectron satellite laser ranging at kilohertz rates. In: SPIE Proceedings Volume 3218, Laser Radar Ranging and Atmospheric Lidar Techniques. London, UK, 24-27 September, pp. 63-77. 
Degnan, J.J, McGarry, J., Dabney, P., Zagwodzki, T., Tierney, M., Weatherley, M., 1998. Design and test of a breadboard interplanetary laser transponder. In: Proc. 11th International Workshop on Laser Ranging. Deggendorf, Germany, 21-25 September, pp. 716-728.

Degnan, J.J., Zayhowski, J.J., 1998. SLR2000 microlaser performance: theory vs experiment. In: Proc. 11th International Workshop on Laser Ranging, Vol. 2. Deggendorf, Germany, 21-25 September, pp. 458-468.

Degnan, J.J., 2000. A detailed analysis of an Earth-Mars laser transponder link. In: Proc. 12th International Workshop on Laser Ranging, Matera, Italy, 13-17 November.

Degnan, J.J., 2001. Photon-counting microlaser rangers, transponders, and altimeters. Surveys in Geophysics 22, 431477.

Degnan, J.J., 2002. Photon-counting multikilohertz microlaser altimeters for airborne and spaceborne topographic measurements. J. Geodynamics 34, 503-549.

Dickey, J.O., Bender, P.L., Faller, J.E., Newhall, X.X., Ricklefs, R.L., Ries, J.G., Shelus, P.J., Veillet, C., Whipple, A.L., Wiant, J.R., Williams, J.G., Yoder, C.F., 1994. Lunar laser ranging: a continuing legacy of the Apollo Program. Science 265, 482-490.

Giorgini, J. Jet Propulsion Laboratory, Pasadena, CA, private communication.

Lemoine, F.J., 2000. NASA Goddard Space Flight Center, Greenbelt, MD 20771, USA, private communication.

Mallama, A., 1998. Earth as seen from Vesta for the transponder proposal, Raytheon STX Corporation Memo to NASA Goddard Space Flight Center, Greenbelt, MD 20771 USA, May 13.

McElroy, J.H., McAvoy, N., Johnson, E.H., Degnan, J.J., Goodwin, F.E., Henderson, D.M., Nussmeier, T.A., Stokes, L.S., Peyton, B.J., Flattau, T., 1977. $\mathrm{CO}_{2}$ LASER communication systems for near Earth space applications. Proc. IEEE 65, 221-251.

RCA Electro-Optics Handbook, 1968. RCA Commercial Engineering, Harrison, NJ.

Schreiber, U., Kawano, N., Yoshino, T., Degnan, J., Nordtvedt, K., Muller, J., Schluter, W., Kunimori, H., 1999. Proc. International Workshop on GEodetic Measurements by the collocation of Space Techniques ON Earth (Gemstone) Communications Research Laboratory, Koganei, Tokyo, Japan, 25-28 January, , pp. 131-136.

Smith, D.E., Zuber, M.T., Solomon, S.C., Phillips, R.J., Head, J.W., Garvin, J.B., Banerdt, W.B., Muhleman, D.O., Pettengill, G.H., Neumann, G.A., Lemoine, F.G., Abshire, J.B., Aharonson, O., Brown, C.D., Hauck, S.A., Ivanov, A.B., McGovern, P.J., Zwally, H.J., Duxbury, T.C., 1999. The global topography of Mars and implications for surface evolution. Science 284, 1495-1503.

Titterton, P., Sweeney, H., Leonard, D., 1998. System/usage impact of operating the SLR2000 at 2 kHz. In: Proc. 11th International Workshop on Laser Ranging, Deggendorf, Germany, 21-25 September, pp. 426-437.

Zissis, G.J. (Ed.), 1993. The Infrared and Electro-optical Systems Handbook, Vol. 1: Sources of Radiation. SPIE Optical Engineering Press, Bellingham, WA, USA (Chapter 3). 